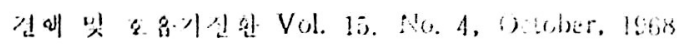

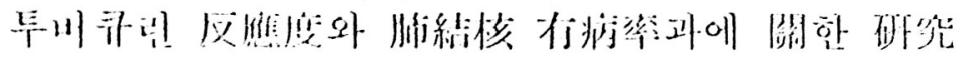

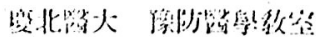

\section{滦疗珠・禁性 萈}

\section{緒䨐}

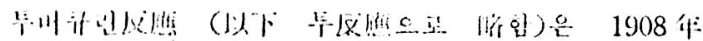

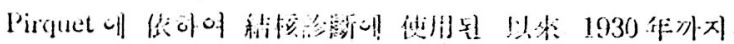

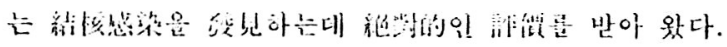

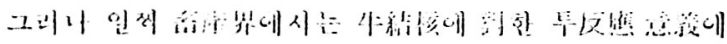

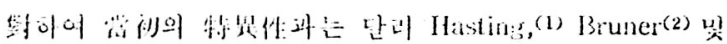

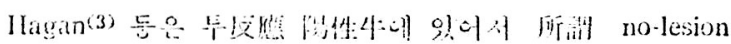

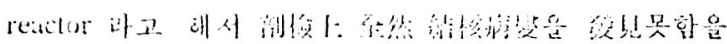

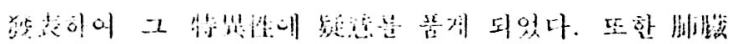

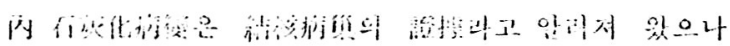

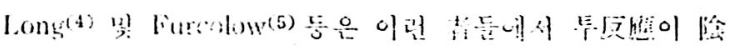

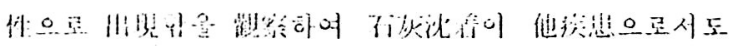

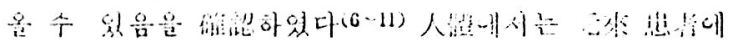

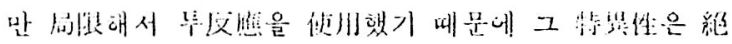

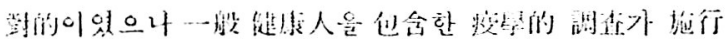

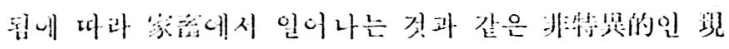

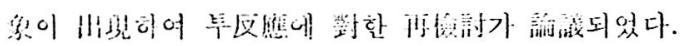

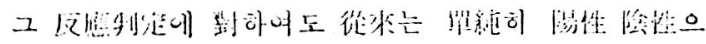

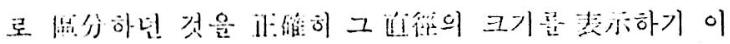

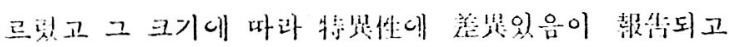
있다. fill Edwards 및 Krohn(12) 동은 $5 \mathrm{~mm}$ 以上 투린

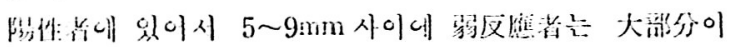

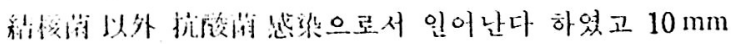

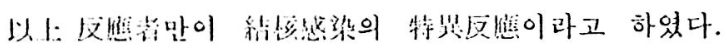

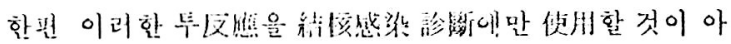

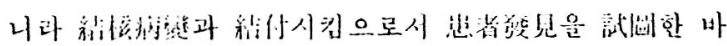
잇으니 새 Stephen (13), Palmer(14) 및 William(15) 등은 便

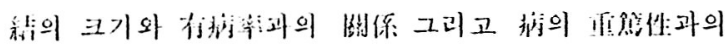

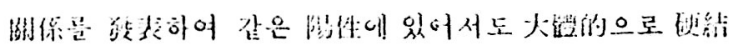

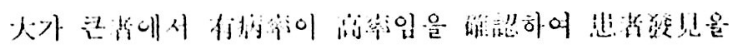

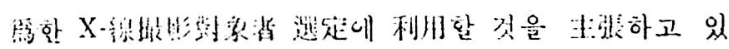

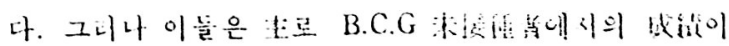

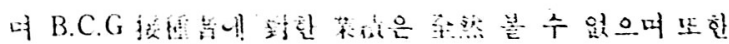

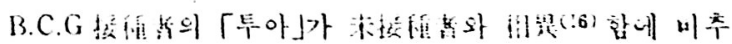

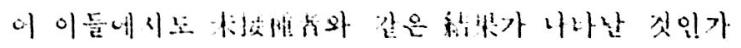
탰배이이다.

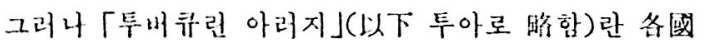

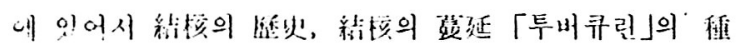

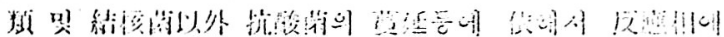

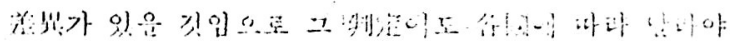

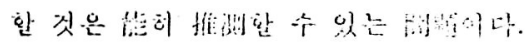

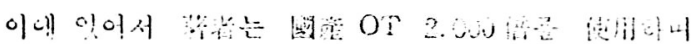

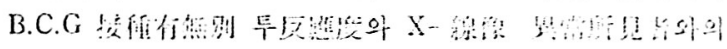

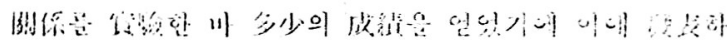
는 바이다.

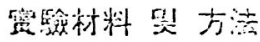

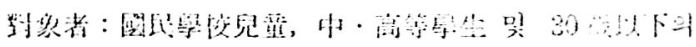

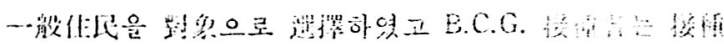

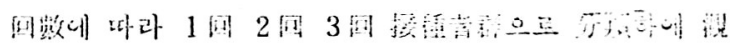
형하였다.

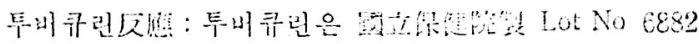

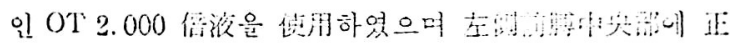

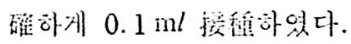

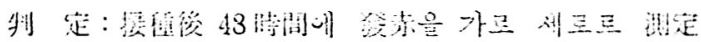

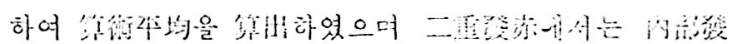

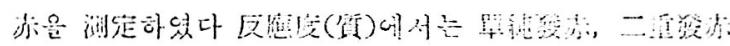

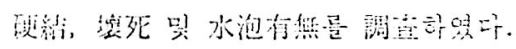

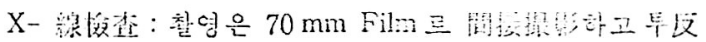

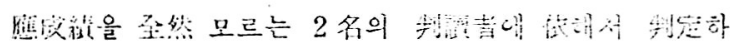
였다.

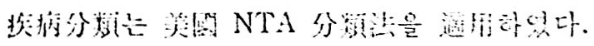

\section{留驗成漬}

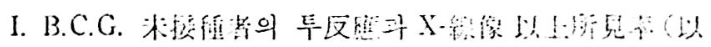

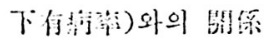

施成, 程

투르.

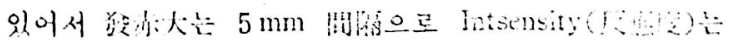

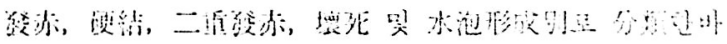

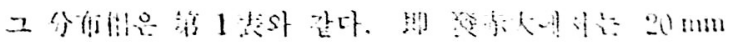

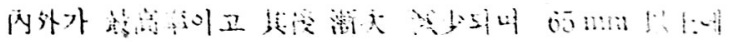

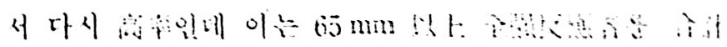
하 배난이아.

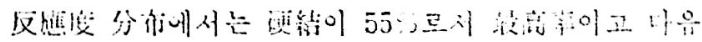

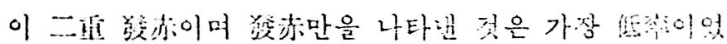
다. 


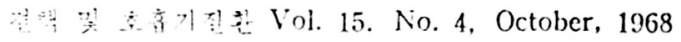

Table 1-a. Corrclation between radiological findings and tuberculin reaction in nonvaccinatees

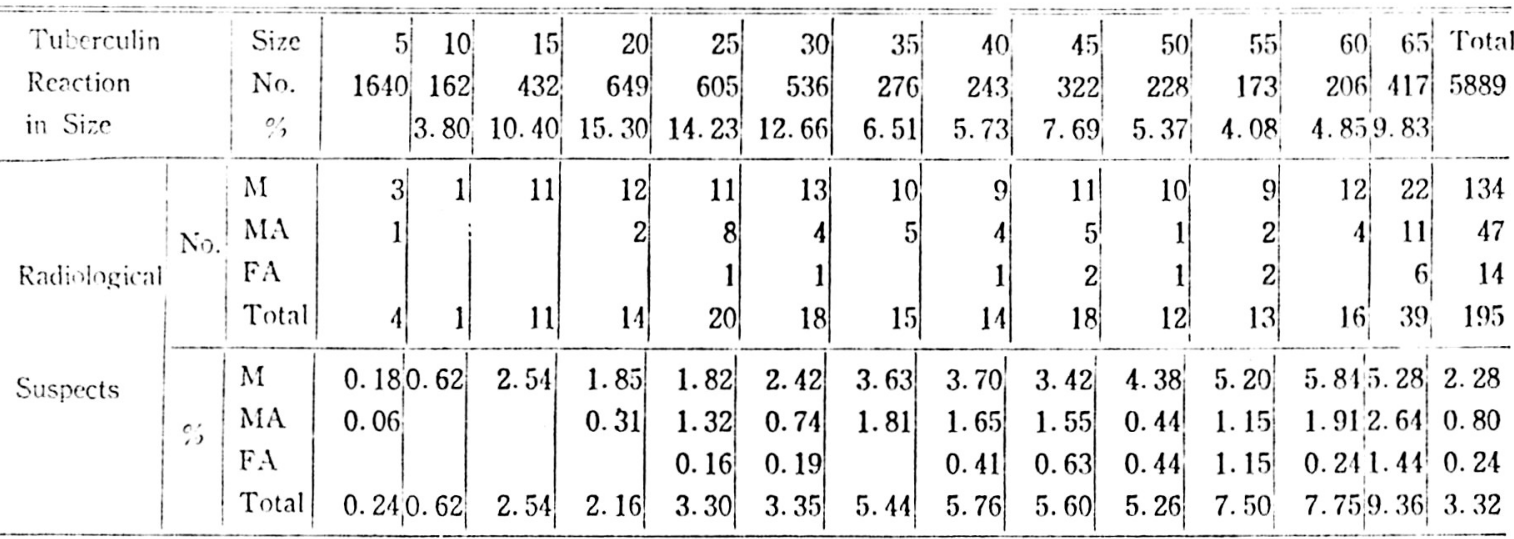

Remarks. Ni; Minimal

MA: Moderate advanced

FA; Far advanced

Toble $1-b \quad$ Correlation between radiological findings and tuberculin reaction in nonvaccinatees

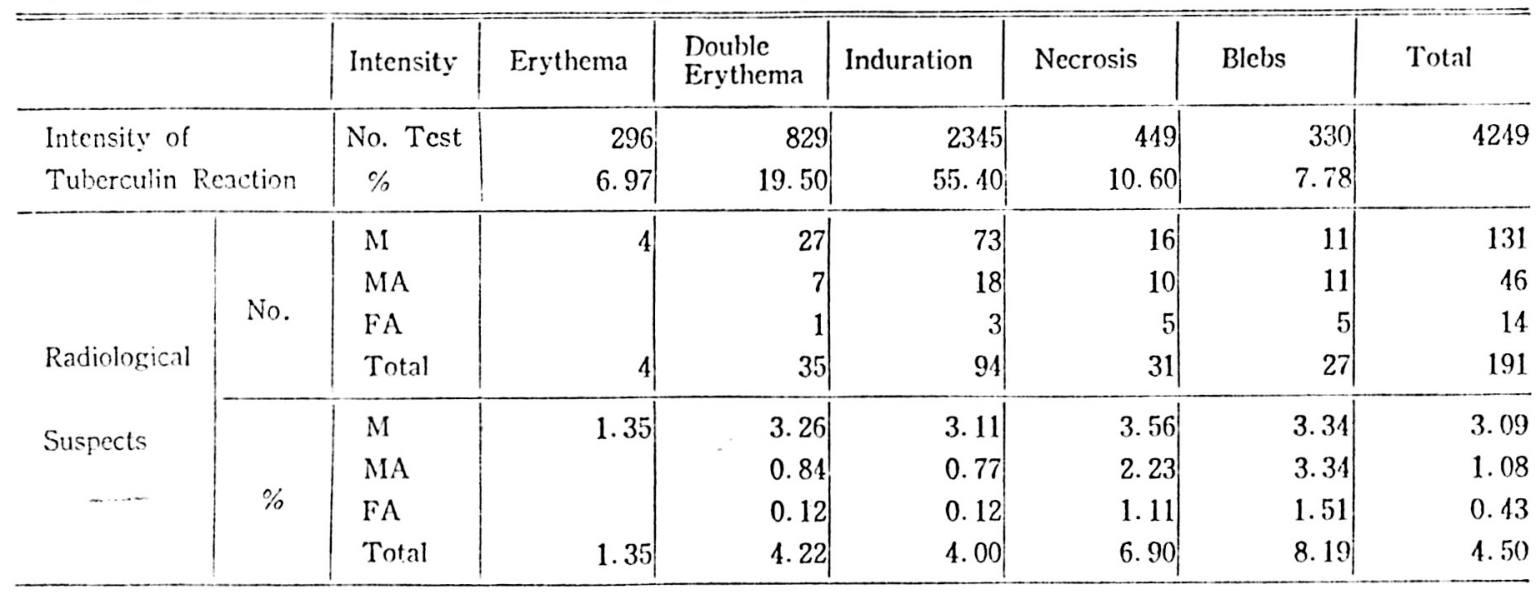

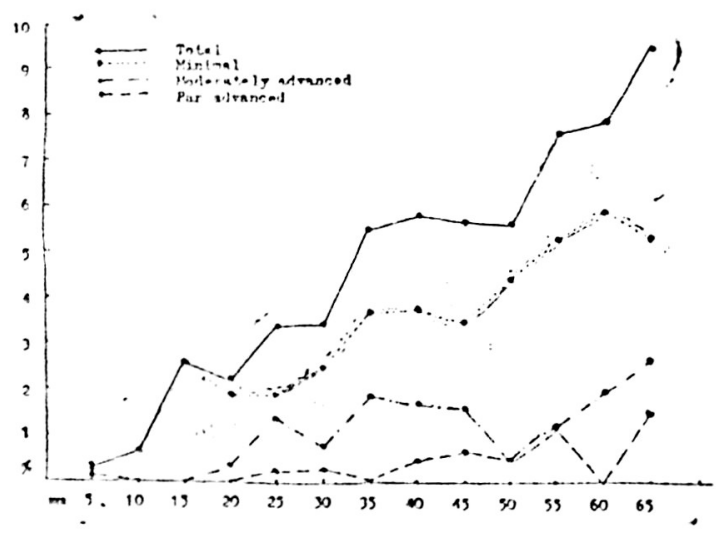

Fis. 1. Correlation between radiengetical findingss and tulerculin reaction innonvaccinalces.

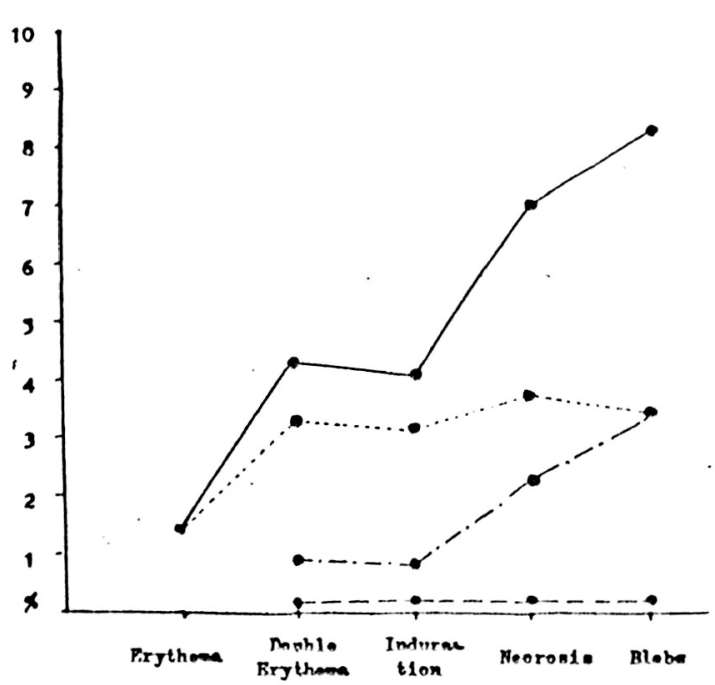

Figg. 2. Correlation belween raclinlengical findings: and tuberculin reaction in nonvarcinate. 
Table 2 a. Correlation between radiological findings and tuberculin reaction by sex

Male

\begin{tabular}{|c|c|c|c|c|c|c|c|c|c|c|c|c|c|c|c|}
\hline \multicolumn{2}{|l|}{$\begin{array}{l}\text { Tuberculin } \\
\text { Reaction } \\
\text { in Size }\end{array}$} & $\begin{array}{l}\text { Size } \\
\text { No. } \\
\%\end{array}$ & \begin{tabular}{r|r|}
527 & 10 \\
93 \\
3. & 84
\end{tabular} \mid & $\begin{array}{r}15 \\
300 \\
12.40\end{array}$ & $\begin{array}{r}20 \\
422 \\
17.40\end{array}$ & $\begin{array}{r}25 \\
343 \\
14.20\end{array}$ & $\begin{array}{r}30 \\
262 \\
10.81\end{array}$ & $\begin{array}{r}35 \\
189 \\
7.81\end{array}$ & $\begin{array}{r}40 \\
118 \\
4.88\end{array}$ & $\begin{array}{r}45 \\
163 \\
6.75\end{array}$ & $\begin{array}{r}50 \\
115 \\
4.75\end{array}$ & $\begin{array}{r}55 \\
87 \\
3.60\end{array}$ & $\begin{array}{r}60 \\
102 \\
4.22\end{array}$ & $\begin{array}{r}6 i \\
220 \\
9.35\end{array}$ & $\begin{array}{l}\text { Potal } \\
3247\end{array}$ \\
\hline \multirow[t]{2}{*}{ Radiological } & No. & $\begin{array}{l}\text { MA } \\
\text { FA } \\
\text { Total }\end{array}$ & \begin{tabular}{l|l|}
2 & 1 \\
1 &
\end{tabular} \mid & 8 & 8 & \begin{tabular}{r|}
6 \\
4 \\
1 \\
11
\end{tabular} & $\begin{array}{l}6 \\
1 \\
1 \\
8\end{array}$ & $\begin{array}{l}7 \\
4\end{array}$ & $\begin{array}{l}5 \\
3\end{array}$ & $\begin{array}{r}6 \\
2 \\
2 \\
10\end{array}$ & $\begin{array}{l}3 \\
1 \\
4\end{array}$ & $\begin{array}{l}5 \\
1 \\
1 \\
7\end{array}$ & $\begin{array}{l}5 \\
2\end{array}$ & $\begin{array}{r}11 \\
4 \\
3 \\
18\end{array}$ & $\begin{array}{r}73 \\
23 \\
9 \\
105\end{array}$ \\
\hline & $\%$ & $\begin{array}{l}\text { M } \\
\text { MA } \\
\text { IAA } \\
\text { Total }\end{array}$ & $\left|\begin{array}{l|l|}0.24 & 1.07 \\
0.12 & \\
0.36 & 1.07\end{array}\right|$ & 2.66 & $\begin{array}{l}1.89 \\
0.21 \\
2.13\end{array}$ & $\begin{array}{l}1.75 \\
1.17 \\
0.29 \\
3.21\end{array}$ & $\left|\begin{array}{l}2.29 \\
0.38 \\
0.39 \\
3.06\end{array}\right|$ & $\begin{array}{l}3.70 \\
2.12 \\
5.82\end{array}$ & $\begin{array}{l}4.24 \\
2.53 \\
6.77\end{array}$ & $\begin{array}{l}3.68 \\
1.23 \\
1.23 \\
6.15\end{array}$ & $\begin{array}{l}2.61 \\
0.87 \\
3.48\end{array}$ & \begin{tabular}{l|}
5.75 \\
1.15 \\
1.15 \\
8.05
\end{tabular} & $\begin{array}{l}4.90 \\
1.95 \\
6.85\end{array}$ & $\begin{array}{l}4.87 \\
1.78 \\
1.30 \\
7.95\end{array}$ & $\begin{array}{l}2.24 \\
0.71 \\
0.28 \\
3.23\end{array}$ \\
\hline
\end{tabular}

Table 2-b. Correlation between radiological findings and tuberculin reaction by sex

\begin{tabular}{|c|c|c|c|c|c|c|c|c|}
\hline & & Intensity & Erythema & $\begin{array}{c}\text { Double } \\
\text { Erythema }\end{array}$ & Induration & Necrosis & Blebs & Total \\
\hline \multicolumn{2}{|c|}{$\begin{array}{l}\text { Intensity of } \\
\text { Tuberculin Reaction }\end{array}$} & $\begin{array}{l}\text { No. } \\
\%\end{array}$ & $\begin{array}{l}160 \\
6.6\end{array}$ & $\begin{array}{r}497 \\
25.5\end{array}$ & $\begin{array}{l}1348 \\
55.7\end{array}$ & $\begin{array}{r}267 \\
11.0\end{array}$ & $\begin{array}{l}148 \\
6.1\end{array}$ & 2420 \\
\hline \multirow[t]{2}{*}{ Radiological } & No. & $\begin{array}{l}\text { M } \\
\text { MA } \\
\text { FA } \\
\text { Total }\end{array}$ & 2 & $\begin{array}{r}17 \\
3 \\
1 \\
21\end{array}$ & $\begin{array}{r}37 \\
9 \\
3 \\
49\end{array}$ & $\begin{array}{r}9 \\
5 \\
3 \\
17\end{array}$ & $\begin{array}{r}6 \\
5 \\
2 \\
13\end{array}$ & $\begin{array}{r}71 \\
22 \\
9 \\
102\end{array}$ \\
\hline & $\%$ & $\begin{array}{l}\text { M } \\
\text { MA } \\
\text { FA } \\
\text { Total }\end{array}$ & .1 .25 & $\begin{array}{l}3.42 \\
0.60 \\
0.20 \\
4.22\end{array}$ & $\begin{array}{l}2.74 \\
0.66 \\
0.22 \\
3.62\end{array}$ & $\begin{array}{l}3.37 \\
1.87 \\
1.12 \\
6.36\end{array}$ & $\begin{array}{l}4.05 \\
3.38 \\
1.35 \\
9.78\end{array}$ & $\begin{array}{l}2.93 \\
0.91 \\
0.37 \\
4.22\end{array}$ \\
\hline
\end{tabular}

Table 2-a Correlation between radiological findings and tuberculin reaction by sex

Female

\begin{tabular}{|c|c|c|c|c|c|c|c|c|c|c|c|c|c|c|c|}
\hline $\begin{array}{l}\text { Tuberculin } \\
\text { Reaction } \\
\text { in Size }\end{array}$ & & $\begin{array}{c}\text { Size } \\
\text { No. } \\
\%\end{array}$ & \begin{tabular}{r|r|}
5 & 10 \\
813 & 69 \\
3.77
\end{tabular} & $\begin{array}{r}15 \\
132 \\
7.23\end{array}$ & $\begin{array}{r}20 \\
227 \\
12.50\end{array}$ & $\begin{array}{r}25 \\
262 \\
14.32\end{array}$ & $\begin{array}{r}30 \\
274 \\
14.95\end{array}$ & $\begin{array}{r}35 \\
87 \\
4.75\end{array}$ & $\begin{array}{r}40 \\
125 \\
6.84\end{array}$ & $\begin{array}{r}45 \\
159 \\
8.70\end{array}$ & $\begin{array}{r}50 \\
113 \\
6.17\end{array}$ & $\begin{array}{r}55 \\
86 \\
4.70\end{array}$ & $\begin{array}{r}60 \\
104 \\
5.70\end{array}$ & $\begin{array}{r}65 \\
191 \\
10.42\end{array}$ & $\begin{array}{l}\text { Total } \\
2012\end{array}$ \\
\hline Radiological & No. & $\begin{array}{l}\text { M } \\
\text { MA } \\
\text { FA } \\
\text { Total }\end{array}$ & 1 & 3 & 5 & 9 & $\begin{array}{l}7 \\
3\end{array}$ & 3 & $\begin{array}{l}4 \\
1 \\
1 \\
6\end{array}$ & $\begin{array}{l}5 \\
3\end{array}$ & $\begin{array}{l}7 \\
1\end{array}$ & 6 & $\begin{array}{r}9 \\
-\quad 9\end{array}$ & \begin{tabular}{r|r|}
11 & \\
7 \\
3 \\
21
\end{tabular} & $\begin{array}{r}61 \\
24 \\
5 \\
90\end{array}$ \\
\hline Suspects & $\%$ & $\begin{array}{l}\mathrm{M} \\
\mathrm{MA} \\
\mathrm{FA} \\
\text { Total }\end{array}$ & $\left|\begin{array}{l}0.12 \\
0.12\end{array}\right|$ & 2.27 & $\begin{array}{l}1.76 \\
0.44 \\
2.20\end{array}$ & $\begin{array}{l}1.90 \\
1.53 \\
3.43\end{array}$ & $\begin{array}{l}2.55 \\
1.09 \\
3.64\end{array}$ & $\begin{array}{l}3.45 \\
1.15 \\
4.60\end{array}$ & $\begin{array}{l}3.20 \\
0.80 \\
0.80 \\
4.80\end{array}$ & $\begin{array}{l}3.15 \\
1.88 \\
5.03\end{array}$ & $\begin{array}{l}6.19 \\
0.89 \\
7.08\end{array}$ & $\begin{array}{l}4.65 \\
1.16 \\
1.16 \\
6.97\end{array}$ & $\begin{array}{l}6.73 \\
1.92 \\
8.63\end{array}$ & $\begin{array}{r}5.70 \\
3.66 \\
1.57 \\
10.99\end{array}$ & $\begin{array}{l}2.30 \\
0.91 \\
0.19 \\
3.40\end{array}$ \\
\hline
\end{tabular}




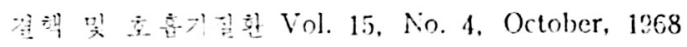

Table 2 b Corrclation betwen radiological findings and tuberculin reaction by scx Femalc

\begin{tabular}{|c|c|c|c|c|c|c|c|c|}
\hline & & Intensity & Erythema & $\begin{array}{l}\text { Double } \\
\text { Erythema }\end{array}$ & Induration & Necrosis & Blcbs & Total \\
\hline \multirow{2}{*}{\multicolumn{2}{|c|}{$\begin{array}{l}\text { Intensity of } \\
\text { Tuhercuin Renction }\end{array}$}} & No. & 136 & 332 & 997 & 182 & 182 & 1829 \\
\hline & & $\%$ & 7.44 & 18.13 & 54.50 & 9.95 & $9.9 i$ & \\
\hline \multirow{4}{*}{ Radiologial } & \multirow{4}{*}{ No. } & M & 2 & 10 & 36 & 7 & 5 & 60) \\
\hline & & $M A$ & & 4 & 9 & 5 & 6 & 21 \\
\hline & & FA & & & & 2 & 3 & 5 \\
\hline & & Total & 2 & 14 & 45 & 14 & 14 & 89 \\
\hline \multirow{4}{*}{ Susperts } & \multirow{4}{*}{ 泊 } & $M$ & 1.47 & 3.02 & 3.62 & 3.85 & 2.74 & 3.29 \\
\hline & & $M A$ & & 1.40 & 0. 90 & 2.95 & 3.31 & 1.32 \\
\hline & & $F A$ & & & & 0.83 & 1.65 & ). 22 \\
\hline & & Total & 1.47 & 4.42 & 4.52 & 7.69 & 7.70 & 482 \\
\hline
\end{tabular}

Table 3 a.

Correhtion bewen rediongrical lindings and reaction to age group 69 (nonvaccinates)

\begin{tabular}{|c|c|c|c|c|c|c|c|c|c|c|c|c|c|c|c|}
\hline Wale & Sizc & 5 & 10 & 15 & $20 !$ & 25 & 30 & 35 & 40 & 4.5 & $50 !$ & 55 & 60) & 65 & rotal \\
\hline Tub. Reactina & No. & 349 & 6 & 19 & 45 & 41 & 55 & 17 & 24 & 31 & 17 & 11 & 14 & $1.3^{\prime}$ & 647 \\
\hline in $S i x c$ & $\%$ & & 2.01 & 6.37 & 15.10 & 13.75 & 18.45 & 5.70 & $8.0 \%$ & 10.40 & 5.70 & 3.69 & 4.69 & 6.05 & \\
\hline & $M$ & 1 & & 1 & & & & & 1 & & & 1 & 1 & & 9 \\
\hline Radiological & $\mathrm{MA}$ & 1 & & & & & & & 1 & & & & & I1 & 5 \\
\hline Suspects & Total & 2 & & 1 & & & & & 2 & 2 & & 1 & 1 & 1 & 14 \\
\hline & 3 & 0.57 & & 5.25 & & & & 2.35 & 8.35 & 6.45 & & 9.10 & 7.15 & 5.5 .5 & 2. 16 \\
\hline Pemale & Size & 5 & 10 & 15 & 20 & 25 & 30 & 35 & 40 & 45 & 50 & 55 & 60 & 65 & \\
\hline Tub. Reactini & No. & 296 & & 15 & 26 & 39 & 50 & 29 & 19 & 29 & 23 & 13 & 27 & 30 & 604 \\
\hline in Size & $\%$ & & 1.32 & 4. 86 & 8.45 & 12.65 & 16.20 & 9.41 & 6.16 & 9.41 & 7.16 & 4.23 & 8.76 & 9.75 & \\
\hline & $M$ & 1 & & & 1 & 1 & & 1 & 2 & & & 1 & 1 & & 9 \\
\hline ....... & $M A$ & & & & & & & & & & & & & & 5 \\
\hline Rathongual & Total & 1 & & & 1 & 1 & & 2 & 2 & & & 1 & 1 & 1 & 14 \\
\hline Surfects & $\%$ & 0.34 & & & 3.85 & 2.57 & & 6.90 & 10.50 & & 11.10 & 7.70 & 3.70 & 3. 33 & 2.32 \\
\hline
\end{tabular}

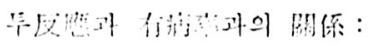

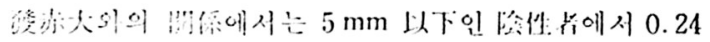
$\%$ 의 1 1

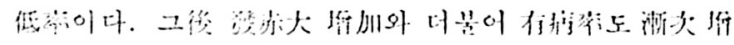

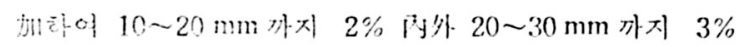

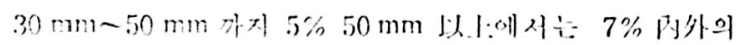

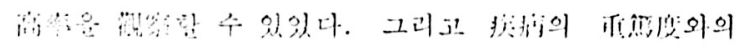

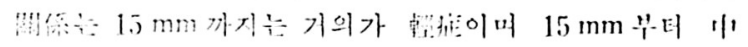

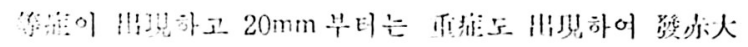

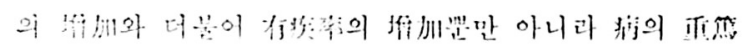
ir.

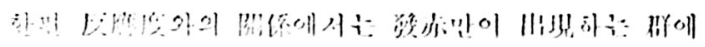

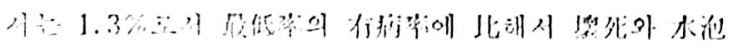

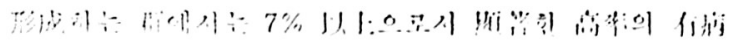

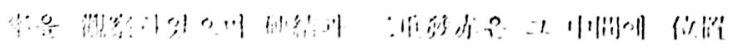

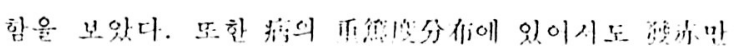

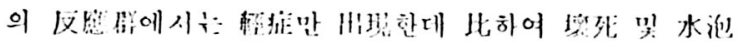

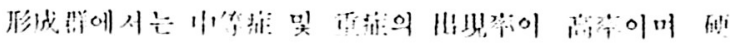

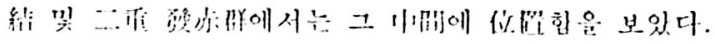

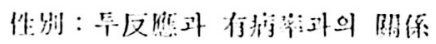

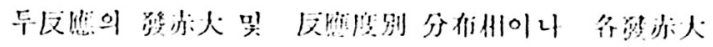

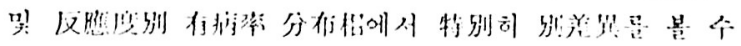
없있 다. ( $p>0.05$ )

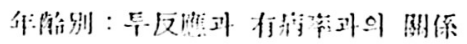

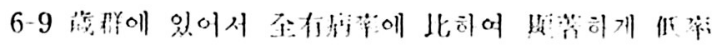

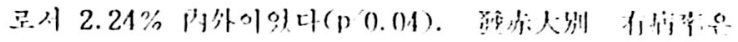

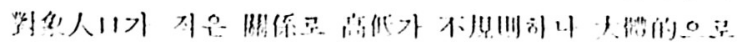

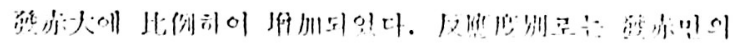

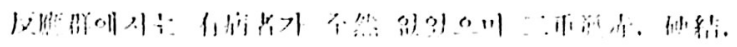

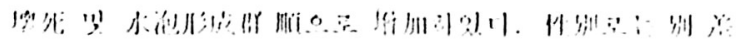




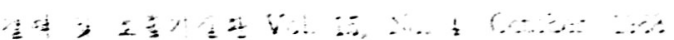

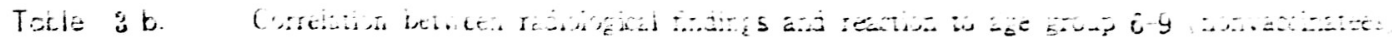

\begin{tabular}{|c|c|c|c|c|c|c|}
\hline & Intencily & Erotienat & $\begin{array}{l}\text { [o,ulde } \\
\text { Erytherna }\end{array}$ & Induration & Necresis & BUbs \\
\hline Maile & No. & 22 & 41 & 166 & 24 & 45 \\
\hline Inte-usity of & $\%$ & 7.38 & 13.75 & 55.60 & 8.05 & 15.10 \\
\hline 'Tulerculin Reaction & il & & 2 & 3 & 1 & 2 \\
\hline & MiA & & 1 & 1 & 1 & 1 \\
\hline Ratiologrical & Total & & 3 & 4 & 2 & 3 \\
\hline Suspects & $\%$ & & 7.32 & 2.41 & 8.35 & 6.68 \\
\hline Fenale & No. & 21 & 38 & 163 & 29 & 57 \\
\hline Intensity of & $\%$ & 6.81 & 12. 32 & 52.95 & 9.41 & 13.50 \\
\hline Tuberculin Reuction & M & : & 1 & 7 & & 2 \\
\hline & MA & & & 1 & 1 & 1 \\
\hline 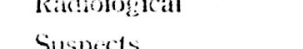 & T'otal & & 1 & 8 & 1 & 3 \\
\hline Suspects & 哭 & & 2.63 & 4.90 & 3.45 & 5.25 \\
\hline
\end{tabular}

Table 4 a. Corration between radiological findings and tuberculin reaction by age group io is (Nonvacinatees)

\begin{tabular}{|c|c|c|c|c|c|c|c|c|c|c|c|c|c|c|c|}
\hline Malc: & Sizc & 5 & 10 & 15 & & & & 35 & 40 & 45 & & 55 & 60 & 65 & Tot: \\
\hline Tulicrculin & No. & 34 & & 10) & 34 & 45 & & 25 & 13 & 22 & 19 & 14 & 25 & 24 & 29 \\
\hline Recation in & $\mathscr{\%}$ & & & 3.80 & 12.91 & 17.01 & 12.20 & 9.51 & 4.95 & 8.35 & 7.25 & 5.32 & 9.50 & 9.15 & \\
\hline Size & $M$ & & & & & & & -1 & & & & 1 & & 1 & \\
\hline Raciologi & $M A$ & & & & & & & & & & & & & & \\
\hline Surects & Total & & & & & 1 & & 1 & 1 & & & 1 & 2 & 1 & \\
\hline & $\%$ & & & & & 2.22 & & 4.00 & 772 & & & 7.15 & 8.00 & 4.17 & 2. \\
\hline cinale & No. & 31 & 2 & 7 & 14 & 20 & 24 & 11 & 9 & 22 & 22 & 19 & 23 & $4 \bar{S}$ & \\
\hline Tub. Reaction & $\%$ & & 0.92 & 3. 21 & 6.42 & 8.03 & 9.64 & 5.05 & 4.12 & 10.10 & 10.10 & 3.71 & 10.51 & 20.62 & \\
\hline in Size & $\mathrm{M}$ & & & i) & & & & & & & & & 1 & & \\
\hline & MA & & & & & & & & & & & & & 2 & \\
\hline Radiological & Total & & & 1 & & & 2 & & & 1 & & & 1 & 0 & 1 \\
\hline Suspects & $\%$ & & & 14.3 & & & 8.34 & & & 4.55 & & & 4.35 & 13.30 & 4. \\
\hline
\end{tabular}

Table $4 \mathrm{~b}$. Correlation between radiological findings and tuberculin reaction by age group 10-13 (nonvacciratees)

\begin{tabular}{|c|c|c|c|c|c|c|}
\hline & Intensity & Erythema & $\begin{array}{l}\text { Double } \\
\text { Erythema }\end{array}$ & Induration & Necrosis & Blebs \\
\hline Male & No. & 17 & 38 & 1.17 & 11 & $\Sigma 0$ \\
\hline Intensity of & $\%$ & 6.45 & 14.45 & 55.90 & 4.18 & 19.05 \\
\hline Tuberculin Reaction & $M$ & & 1 & 3 & & 1 \\
\hline & MA & & & 1 & & 1 \\
\hline Raidiological & loutal & & .1 & 4 & & 2 \\
\hline Suspects & $\%$ & & 2.63 & 2.72 & & 4.00 \\
\hline Female & No. & 12 & 37 & 104 & 10 & 55 \\
\hline Intensity of & $\%$ & 5.50 & 17.00 & 47.70 & 4.58 & $25 \cdot 20$ \\
\hline Tuberculin Reaction & M & & 2 & 3 & 2 & 1 \\
\hline & $\mathrm{MA}$ & & & 1 & & 2 \\
\hline Radiological & Total & & 2 & 4 & 2 & 3 \\
\hline Suspects & $\%$ & & 5.41 & 3.85 & 2.00 & 5.15 \\
\hline
\end{tabular}




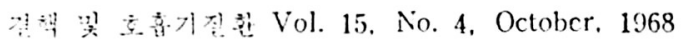

Tabie 5 a. Correlation betwecn radiological findings and tuberculin reaction by age group 14-16 (Nonvaccinatces)

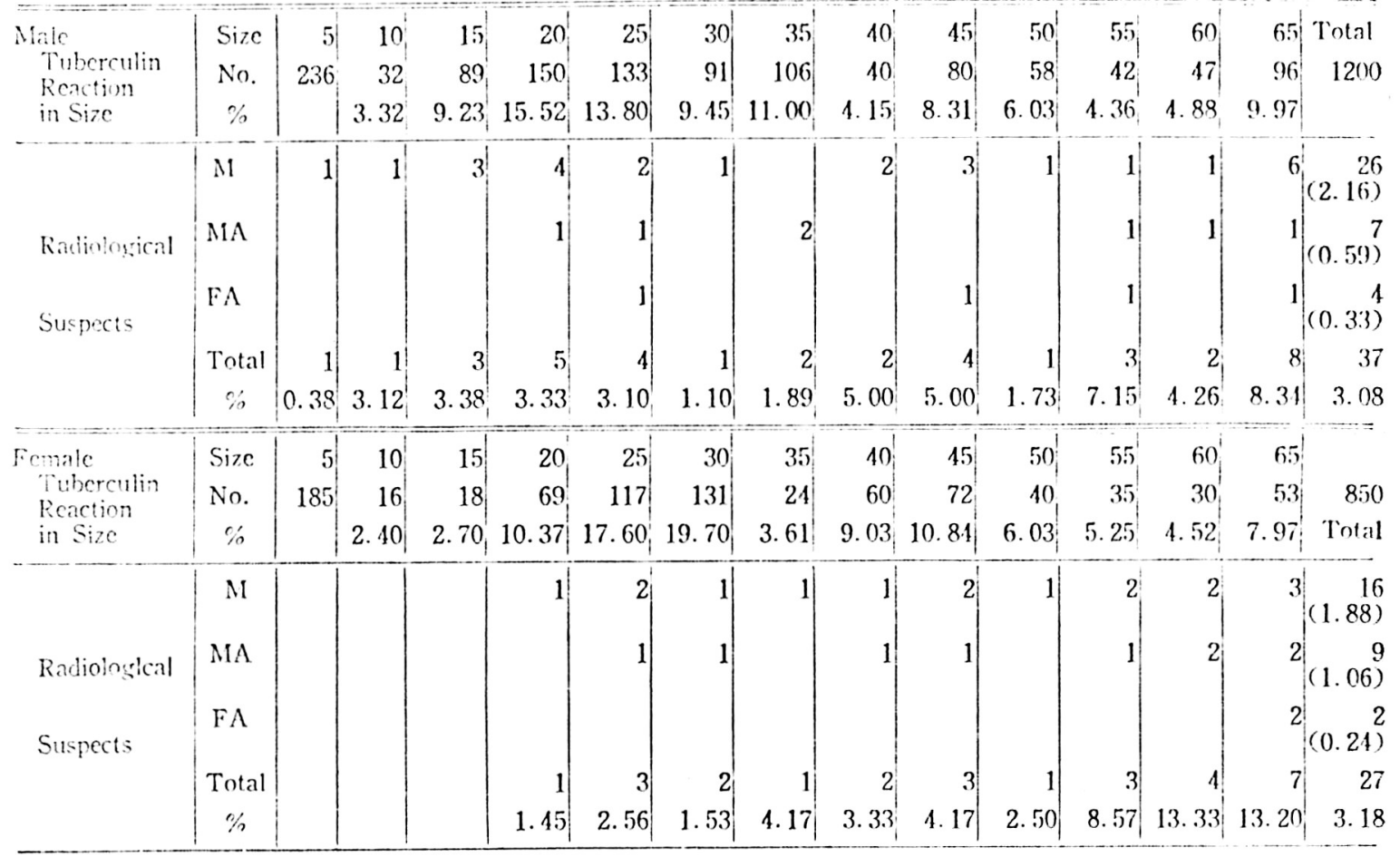

Toble 5-b. Correlation betwecn radiological findings and tuberculin reaction by age group 14-16 (nonvaccinatces)

\begin{tabular}{|c|c|c|c|c|c|c|}
\hline & Intcnsity & Erythema & $\begin{array}{l}\text { Double } \\
\text { Erythema }\end{array}$ & Induration & Necrosis & Blcbs \\
\hline \multirow{5}{*}{$\begin{array}{l}\text { Male } \\
\text { Intersity of } \\
\text { Tuberculin Reaction }\end{array}$} & No. & 18 & 289 & 453 & 153 & 21 \\
\hline & $\%$ & 4. 98 & 30.00 & 47.00 & 15.90 & 2.18 \\
\hline & M & 1 & 7 & 12 & 5 & \\
\hline & MA & & 0 & 3 & 3 & 1 \\
\hline & FA & & 1 & 1 & 1 & 1 \\
\hline \multirow{2}{*}{$\begin{array}{l}\text { Ratiological } \\
\text { Suppets }\end{array}$} & Total & 1 & 8 & 16 & 9 & 2 \\
\hline & $\%$ & 2.09 & 2.77 & 3. 18 & 5.88 & 10.50 \\
\hline \multirow{5}{*}{$\begin{array}{l}\text { Female } \\
\text { Intensity of } \\
\text { Tuberculin Reaction }\end{array}$} & No. & 45 & 169 & 343 & 72 & 36 \\
\hline & $\%$ & 6.78 & 25.40 & 51.60 & 10.81 & 5.42 \\
\hline & $M$ & 1 & 3 & 9 & 2 & 1 \\
\hline & $M \Lambda$ & & 2 & 3 & 2 & 2 \\
\hline & $\mathrm{F} \wedge$ & & & & 1 & 1 \\
\hline \multirow{2}{*}{$\begin{array}{l}\text { Radiongical } \\
\text { Suncets }\end{array}$} & Total & 1 & 5 & 12 & 5 & 4 \\
\hline & $\%$ & 2.22 & 2.96 & 3.50 & 6.94 & 11.10 \\
\hline
\end{tabular}

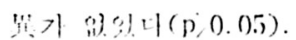

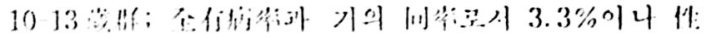

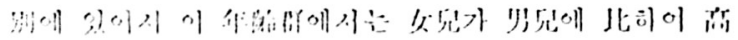
and $4(0,0.0)$.

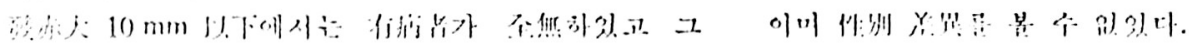

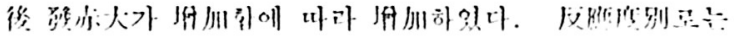

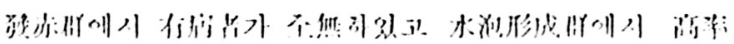
이있다.

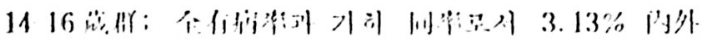
$-16 \cdots$ 
Table 6 a. Corselation lectiwesn radiosegical findings and tuberculin reaction by age group $17 \cdot 19$ (nomvaccinatees)

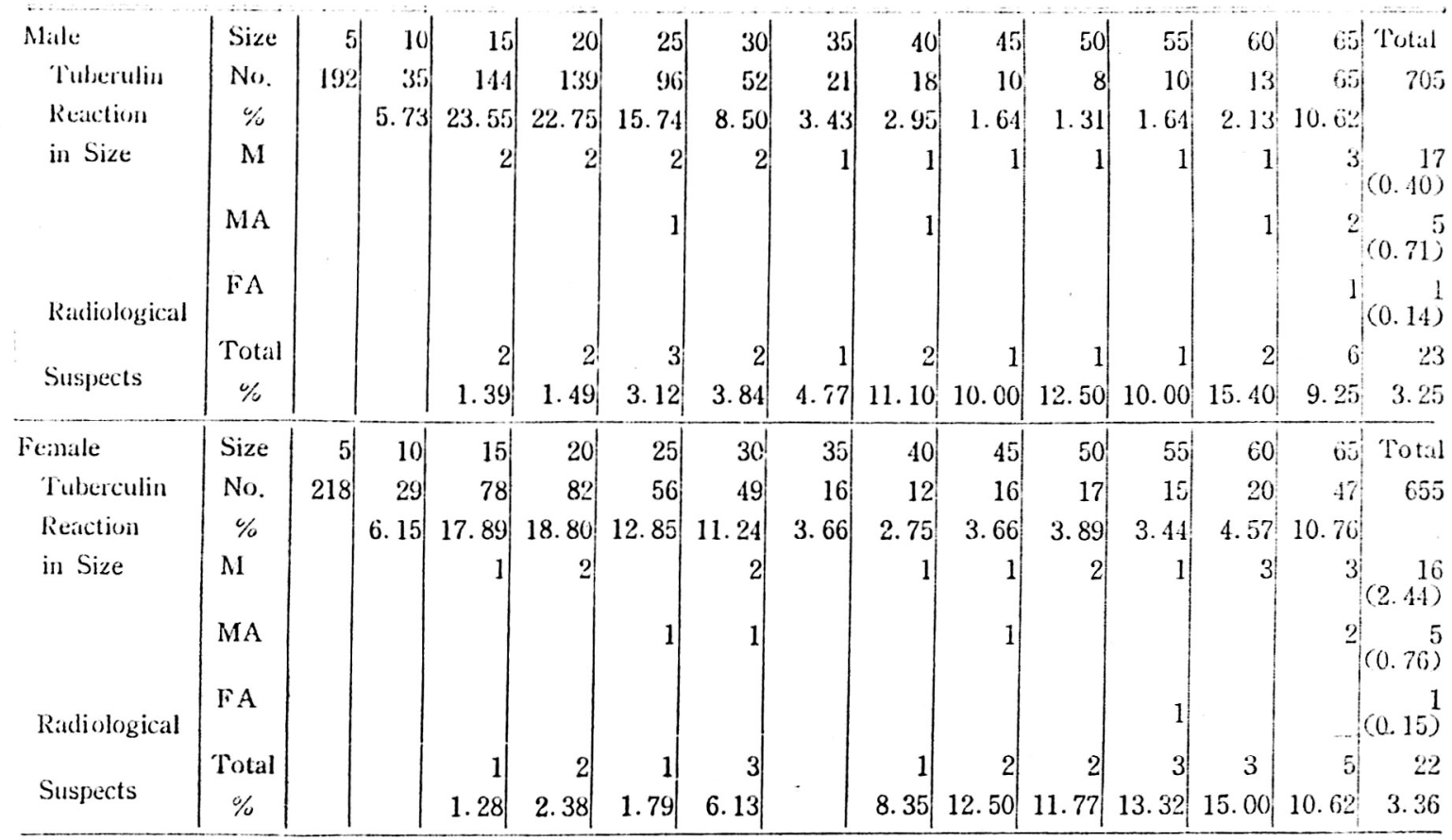

Table 6-b.

Correlation between radiological findings and tuberculin reaction by age group 17-19 (nonvaccinatees)

\begin{tabular}{l|c|r|r|r|r|r}
\hline & Intensity & Erythema & $\begin{array}{c}\text { Double } \\
\text { Erythema }\end{array}$ & Induration & Necrosis & Elebs \\
\hline Male & No. & 63 & 81 & 382 & 69 & 16 \\
Intensity Tuberculin & $\%$ & 10.30 & 13.83 & 62.50 & 11.30 & 2.62 \\
Reaction & $\mathrm{M}$ & 1 & 3 & 9 & 1 & 1 \\
& $\mathrm{MA}$ & & 1 & 2 & 1 & 1 \\
& $\mathrm{FA}$ & & & & 1 & \\
Radiological & Total & 1 & 4 & 11 & 4 & 3 \\
Suspects & $\%$ & 1.59 & 4.34 & 2.98 & 5.80 & 13.70 \\
\hline Female & No. & 38 & 52 & 267 & 67 & 13 \\
Intensity Tuberculin & $\%$ & 8.70 & 11.90 & 61.10 & 15.35 & 2.98 \\
Reaction & $\mathrm{M}$ & & 2 & 11 & 3 & 1 \\
& $\mathrm{MA}$ & & 1 & 1 & 2 & 0 \\
Radiclogical & Totil & & 3 & 12 & 5 & 2 \\
Suspects & $\%$ & 5.76 & 4.50 & 7.45 & 15.40 \\
\hline
\end{tabular}

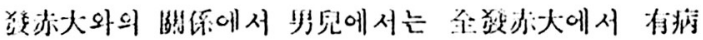

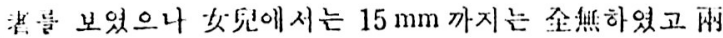

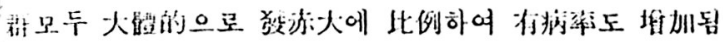

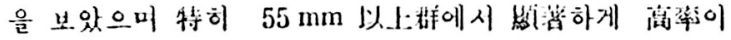

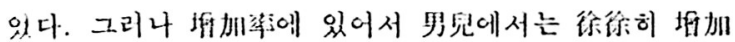

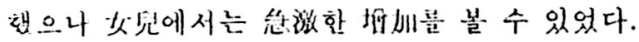

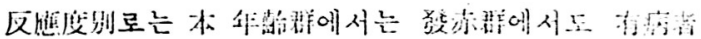

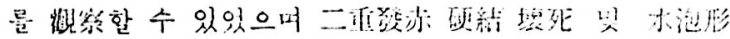

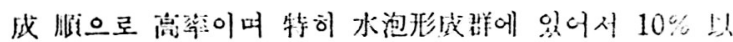

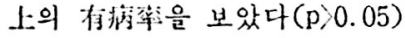

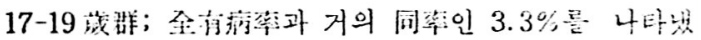

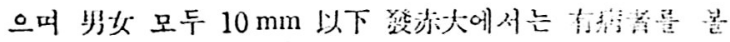




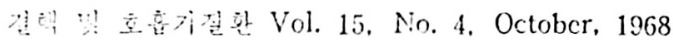

Table $i$-a. Correlation between radiological findings ard tuberculin reaction by age group $20 \&$ over (nonvaccinatecs)

\begin{tabular}{|c|c|c|c|c|c|c|c|c|c|c|c|c|c|c|}
\hline Maic & Size & 5 & 10 & 15 & 20 & 25 & 30 & 35 & 40 & 45 & 50 & 55 & 60 & 65 Total \\
\hline Tuberculin & No. & 16 & 20 & 38 & 54 & 28 & 32 & 20 & 23 & 20 & 13 & 10 & 3 & 300 \\
\hline Ranction in & $\%$ & & 7.05 & 13.38 & 19.00 & 9.86 & 11.27 & 7.05 & 8. 10 & 7.05 & 4.53 & 3.52 & 1.0. & 8.10 \\
\hline $5 i z 0$ & $M$ & & & & & & & & & & 1 & 1 & & $1 \mid \begin{array}{r}16 \\
(5.33)\end{array}$ \\
\hline & $M A$ & & & & & 1 & & & & 1 & & & & $\begin{array}{r}4 \\
(1.33)\end{array}$ \\
\hline Radinlogical & Fil & & & & & & 1 & & & 1 & & & & $1(1.33)^{4}$ \\
\hline & Total & & & 2 & 2 & 3 & 5 & & & $?$ & 2 & 1 & & 24 \\
\hline Suspects & $\%$ & & & 5.26 & 3.7 & 10.7 & 15.60 & 15.00 & 4. 35 & 15.00 & 15.40 & 10.00 & & $8.70 \quad 8.00$ \\
\hline Fcmalc & Sizc & 5 & 10 & 15 & 20 & 25 & $30 !$ & 35 & 40 & 45 & 50 & 55 & $60^{\prime}$ & 65 Total \\
\hline Tuberculn & No. & 83 & 14 & 14 & 36 & 30 & 20 & 7 & 23 & 20 & 11: & 4 & 4 & 16 \\
\hline Feaction in & $\because$ & & 6.97 & 6.97 & 17.90 & $14.90^{\circ}$ & 7.95 & 3.43 & 12.45 & 9.95 & 5.67 & $1.90^{\prime}$ & 1.80 & 7.95 \\
\hline Size & $M$ & & & & & & 2 & & & 1 & & & & $1 \quad \begin{array}{r}10 \\
(3.52)\end{array}$ \\
\hline & $\mathrm{MA}$ & & & & 1 & & 1 & & & & & & & $(1.41)^{4}$ \\
\hline Radiongical & $\mathrm{FA}$ & & & & & & & & 1 & & & & & ${ }^{1}(0.71)^{2}$ \\
\hline & Total & & & 1 & 1 & 4 & & 1 & 1 & 2 & 1 & & & $2 \quad 16$ \\
\hline Susp? & $\%$ & & & 7.15 & 2.78 & 13.30 & 15.00 & 14.29 & $4.0 \%$ & 10.00 & 0.10 & & & $12.08 \quad 5.61$ \\
\hline
\end{tabular}

Tcbis $7-\mathrm{b}$. Correlation between radiological findings and tuberculin reaction by age group $20 \&$ over (nonvaccinatees)

\begin{tabular}{|c|c|c|c|c|c|c|}
\hline & Intensity & Erythema & $\begin{array}{l}\text { Double } \\
\text { Erythema }\end{array}$ & Inrluration & Necrosis & Ilebs \\
\hline \multirow{7}{*}{$\begin{array}{l}\text { Nale } \\
\text { Intenty Tuberculin } \\
\text { Reation }\end{array}$} & No. & 10 & 18 & .200 & 10 & 16 \\
\hline & $\%$ & 3.52 & 16.90 & 70.50 & 3.52 & 5.64 \\
\hline & M & & 4 & 10 & 1 & 1 \\
\hline & $M A$ & & 1 & 2 & & 1 \\
\hline & $\mathrm{FA}$ & & & 2 & 1 & 1 \\
\hline & Total & & 5 & 14 & 2 & 3 \\
\hline & $\%$ & & 10.12 & 7. 00 & 20.00 & 18.80 \\
\hline \multirow{7}{*}{$\begin{array}{l}\text { Fomaic } \\
\text { intensity Tuberculin } \\
\text { Reaction }\end{array}$} & No. & 20 & 36 & 120 & 4 & 21 \\
\hline & $\%$ & 9.95 & 17.90 & 59.90 & 1.96 & 10.43 \\
\hline & M & 1 & 2 & 6 & & 1 \\
\hline & MA & & 1 & 3 & & \\
\hline & $\mathrm{F} \Lambda$ & & & & 1 & 1 \\
\hline & Total & 1 & 3 & 9 & 1 & 2 \\
\hline & $\%$ & 5.00 & 8.35 & 7.48 & 25.00 & 9.55 \\
\hline
\end{tabular}

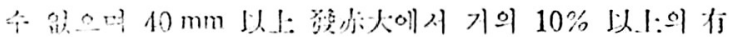

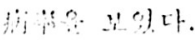

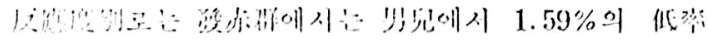

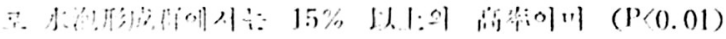
ㄱ. $\because 4(6 ; 1,1)$.

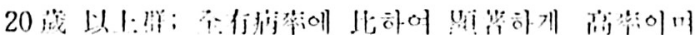

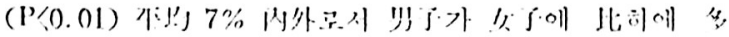

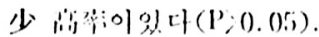

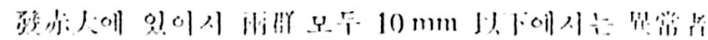

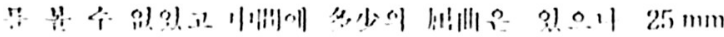

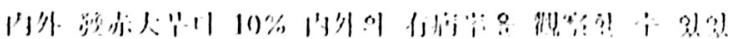




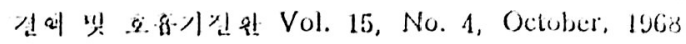

Table $8 \mathrm{a}$.

Correlation between radiological findings and tuberculin reaction in vaccinatees

\begin{tabular}{|c|c|c|c|c|c|c|c|c|c|c|c|c|c|c|c|}
\hline Tuberculin & Size & 5 & 10 & 15 & 20 & 25 & 30 & 35 & 40 & 45 & 50 & 5.5 & 60 & 65 & Total \\
\hline & No. & 1984 & 553 & 937 & 718 & 455 & 223 & 160 & 100 & 148 & 137 & 93 & 132 & 126 & 5816 \\
\hline Retiction in & $\%$ & & 14.45 & 24.45 & 18.72 & 11.87 & 5.83 & 4.18 & 2.60 & 3.87 & 3.58 & 2.42 & 3.44 & 4.59 & \\
\hline Size & $M$ & 6 & 6) & 16 & 12 & 9 & 1 & 2 & & 2 & 1 & & & 2 & 57 \\
\hline & $\mathrm{MA}$ & 1 & & 2 & & & & 1 & & 0 & 1 & & 2 & & 14 \\
\hline & $\mathrm{FA}$ & & & & & & & & & & & & & 1 & 1 \\
\hline & Total & 7 & & 18 & 16 & 10 & 1 & 3 & & 2 & 2 & & 2 & 5 & 72 \\
\hline & $M$ & 0.30 & 1.08 & 1.71 & 1.67 & 1.97 & 0.47 & 1.25 & & 1.35 & 0.73 & & & 1.59 & 0.98 \\
\hline Radiological & $\mathrm{MA}$ & 0.05 & & 0.21 & 0.56 & 0.22 & & 0.62 & & & 0.73 & & 1.52 & 1.59 & 0.24 \\
\hline & FA & & & & & & & & & & & & & 0.79 & 0.01 \\
\hline Suspects & Total & 0.35 & 1.08 & 1.92 & 2.23 & 2.19 & 0.47 & 1.87 & & 1.35 & 1.46 & & 1.52 & 3.97 & 1.24 \\
\hline
\end{tabular}

Table 8 b.

Correlation between radiological findings and tuberculin reaction in vaccinatees.

\begin{tabular}{|c|c|c|c|c|c|c|c|}
\hline & Intensity & Erythema & $\begin{array}{l}\text { Double } \\
\text { Erythema }\end{array}$ & Induration & Necrosis & Blebs & Total \\
\hline \multirow{2}{*}{ Intensity } & No. & 1582 & 575 & 1405 & 143 & 121 & 3825 \\
\hline & 20 & 41.30 & 15.00 & 36.75 & 3.89 & 3.16 & \\
\hline Tuberculin & $\mathrm{M}$ & 22 & 6 & 21 & 1 & & .50 \\
\hline \multirow[t]{4}{*}{ Reaction } & MA & 4 & 1 & 4 & 4 & 1 & 14 \\
\hline & $\mathrm{FA}$ & & & & 1 & & 1 \\
\hline & Tutal & 26 & 7 & 25 & 6 & 1 & 65 \\
\hline & $\mathrm{M}$ & 1.39 & 1.04 & 1.49 & 0.67 & & 1.30 \\
\hline \multirow{2}{*}{ Radiological } & $\mathrm{MA}$ & 0.25 & 0.17 & 0.29 & 2. 69 & 0.83 & 0.37 \\
\hline & $\mathrm{FA}$ & & & & 0.67 & & 0.03 \\
\hline Suspects & Total & 1.64 & 1.22 & 1.78 & 4.03 & 0.83 & 1.70 \\
\hline
\end{tabular}

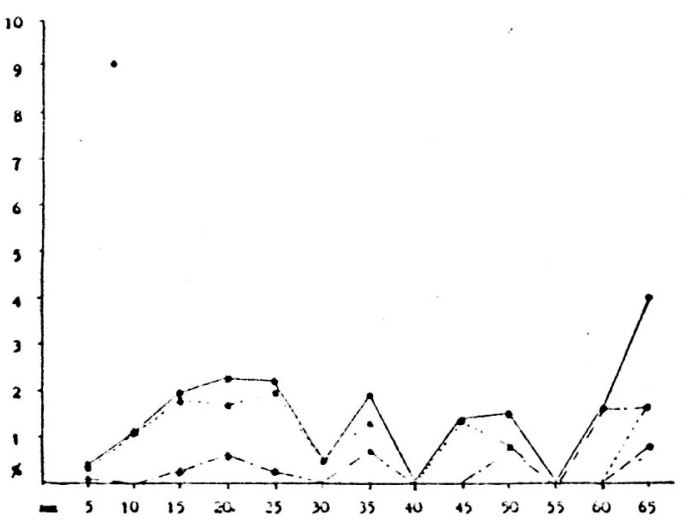

Fig. 3. Correlation between radiological fingings and tuberculin reaction in vaccialatees.

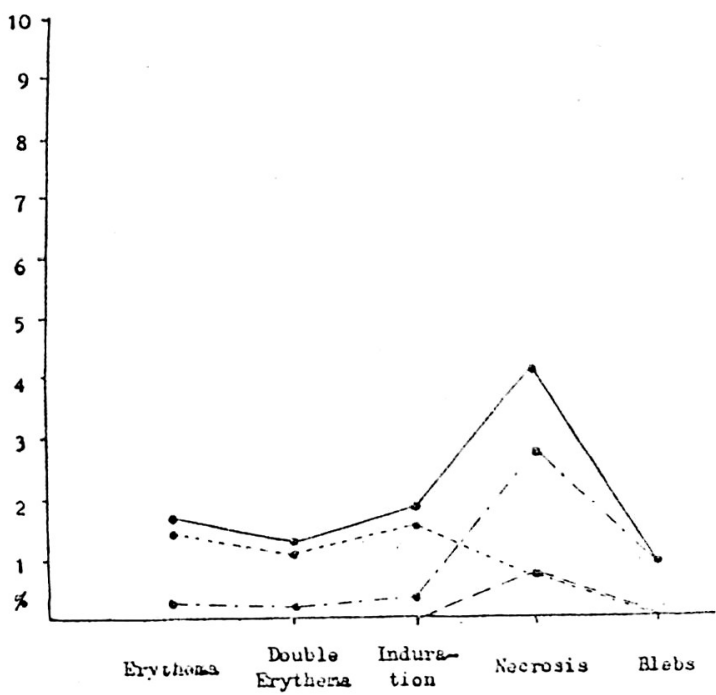

Fib. 4. Correlation between radiological findings and tuberculin reaction in vaccinates. 


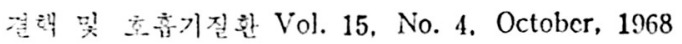

Table 9 a. Correlation betwecn radiological findings and tuberculin reaction in vaccinatecs by sex

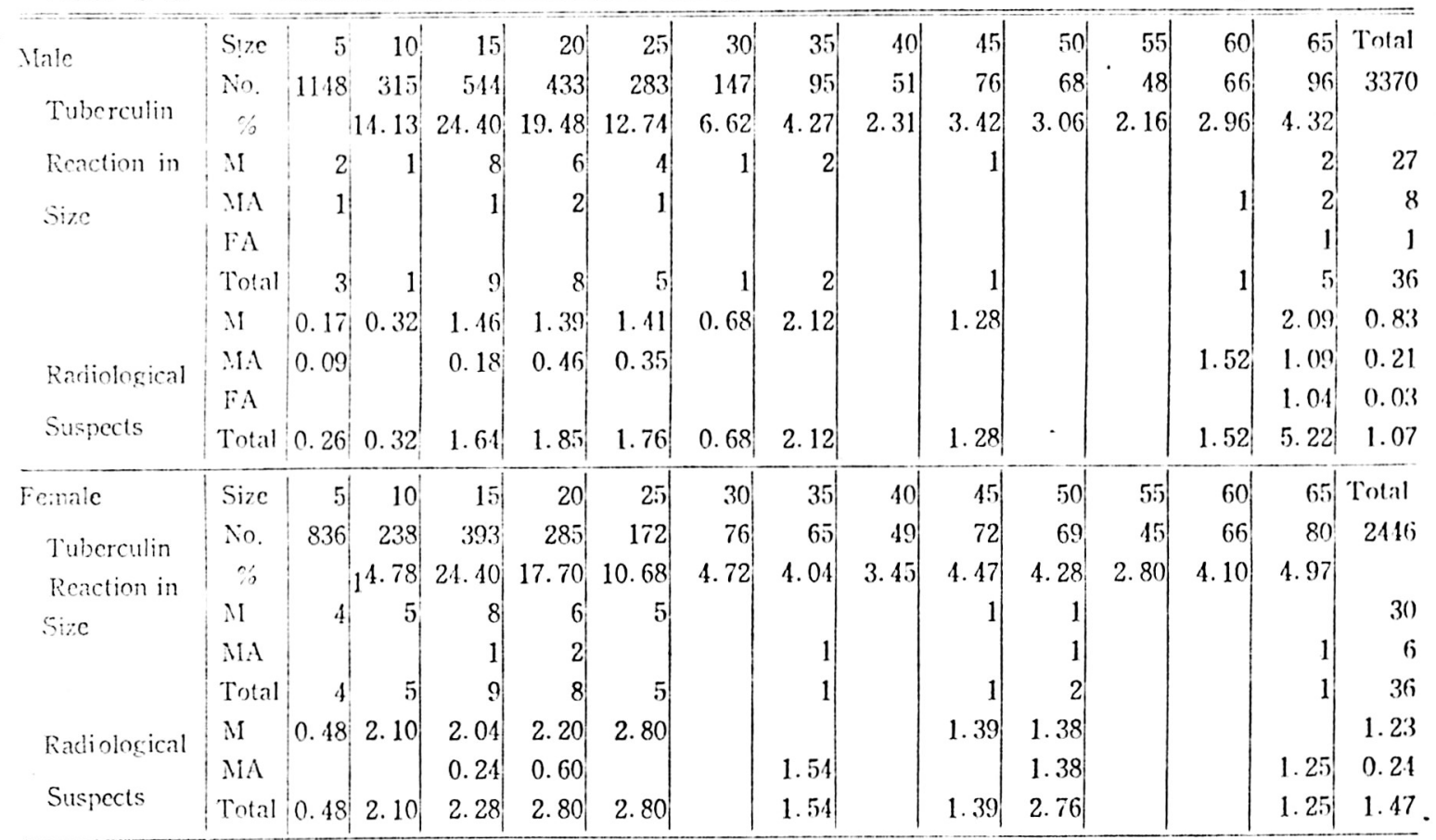

Table $9 \mathrm{~b}$ Correlation between radiological findings and tuberculin reaction in raccinalecs by sex

\begin{tabular}{|c|c|c|c|c|c|c|}
\hline & Intensity & Erythema & $\begin{array}{l}\text { Double } \\
\text { Erythema }\end{array}$ & Induration & Necrosis & Blcbs \\
\hline \multirow{7}{*}{$\begin{array}{l}\text { Nale } \\
\text { Intensity } \\
\text { Tuberculin Reaction }\end{array}$} & No. & 911 & 317 & 817 & 87 & 60 \\
\hline & $\%$ & 40.90 & 15.57 & 36.70 & 3.91 & 1.69 \\
\hline & $M$ & 11 & 2 & 11 & 1 & \\
\hline & MA & 2 & 1 & 1 & 3 & \\
\hline & FA & & & & 1 & \\
\hline & Total & 13 & 3 & 12 & 5 & \\
\hline & $\mathrm{M}$ & 1.21 & 0.58 & 1.35 & 1.15 & \\
\hline \multirow{2}{*}{ Radinlogsica! } & $M A$ & 0.22 & 0.24 & 0.12 & 3.45 & \\
\hline & & & & & 1.15 & \\
\hline Suspects & Total & 1.43 & 0.82 & 1.47 & 5.75 & \\
\hline \multirow{3}{*}{$\begin{array}{l}\text { Fomale } \\
\text { Intensity Tulycrculin }\end{array}$} & $N()$. & 671 & 228 & 588 & 62 & 61 \\
\hline & $\%$ & 41.70 & 14.15 & 36.50 & 3.85 & 3.79 \\
\hline & $M$ & 11 & 4 & 10 & & \\
\hline \multirow[t]{2}{*}{ Reaction } & $M A$ & 2 & & 3 & 1 & 1 \\
\hline & Total & 13 & 4 & 13 & 1 & 1 \\
\hline \multirow{2}{*}{ Radiromsical } & M & 1.61 & 1.75 & 1.70 & 5.61 & 1.64 \\
\hline & MA & 0.30 & & 0.51 & & \\
\hline Suepects & Total & 1.94 & 1.75 & 2.21 & 5.61 & 1.64 \\
\hline
\end{tabular}

$=$.

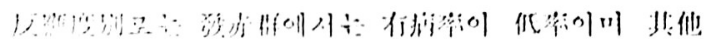

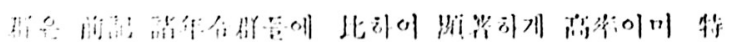

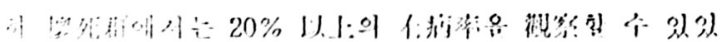

다 $(\mathrm{P} \backslash 0.05)(7-\mathrm{a}, \mathrm{h})$.

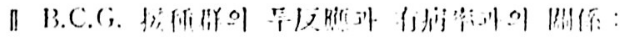

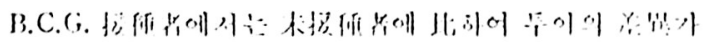

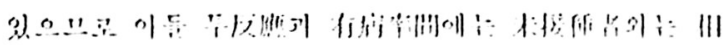




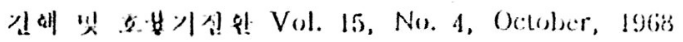

Table 10 1 a. Correlation between radiological findings and tuberculin reaction in vaccinatees once.

\begin{tabular}{|c|c|c|c|c|c|c|c|c|c|c|c|c|c|c|c|}
\hline iculin & Sizc & & 10 & 15 & 20 & 25 & 30 & 35 & & 45 & & 55 & & 65 & lowa! \\
\hline Reaction in & No. & 1022 & 171 & 189 & 153 & 123 & 87 & 56 & 46 & 51 & 51 & 41 & 50 & 78 & 2118 \\
\hline Size & $\%$ & & 15.10 & 17.25 & 13.95 & 11.23 & 7.95 & 5.12 & 4.20 & 4.65 & 4.65 & 3.74 & 4.56 & 7.13 & \\
\hline Des & $\mathrm{M}$ & 4 & & 7 & 2 & & 1 & 1 & & & & & & & 19 \\
\hline & MA & 1 & & 1 & 2 & & & 1 & & 1 & & & & & i \\
\hline & Total & & & 8 & 4 & 2 & 1 & 2 & & 1 & & & & & 25 \\
\hline Radiological & $\mathrm{M}$ & 0.39 & 1.17 & 3.70 & 1.32 & 1.62 & 1.15 & 1.79 & & 1.96 & & & & & 0.90 \\
\hline & MA & 0.10 & & 0.53 & 1.31 & & & 1.79 & & & & & & & 0.28 \\
\hline Sus! & Total & 0.49 & 1.17 & 4.23 & 2.63 & 1.62 & 1.15 & 3.58 & & 1.96 & & & & & 1.18 \\
\hline
\end{tabular}

Table 10-1-b. Correlation between radiological findings and tuberculin reaction in vaccinatees once.

\begin{tabular}{|c|c|c|c|c|c|c|c|}
\hline & Intensity & Erythema & $\begin{array}{c}\text { Double } \\
\text { Erythema }\end{array}$ & Induration & Necrosis & Blebs & Tutal \\
\hline \multirow{5}{*}{$\begin{array}{l}\text { Intensity } \\
\text { Tuberculin } \\
\text { Reaction }\end{array}$} & No. & 457 & 150 & 385 & 47 & 57 & 1096 \\
\hline & $\%$ & 41.60 & 13.69 & 35.20 & 4.28 & 5.20 & \\
\hline & $\mathrm{M}$ & 9 & 1 & 5 & & & 15 \\
\hline & $\mathrm{MA}$ & 1 & & 3 & & 1 & 5 \\
\hline & Total & 10 & 1 & 8 & & 1 & 20 \\
\hline \multirow{2}{*}{ Radiological } & $\mathrm{M}$ & 1.97 & 0.67 & 1.30 & & & 1.37 \\
\hline & $\mathrm{MA}$ & 0.22 & & 0.52 & & 1.76 & 0.45 \\
\hline Suspects & Total & 2.19 & 0.67 & 1.82 & & 1.76 & 1.82 \\
\hline
\end{tabular}

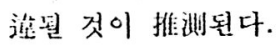

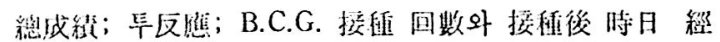

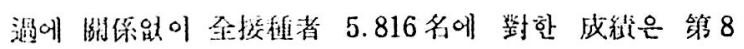

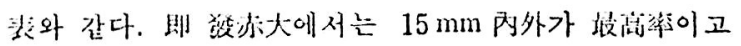
다음이 $20 \mathrm{~mm}$ 이며 罜後渐次 做少하여 $30 \mathrm{~mm}$ 以.1은 $25 \%$ 队外에 不過하였다.

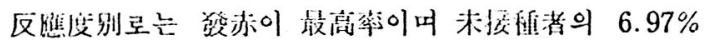

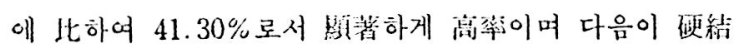

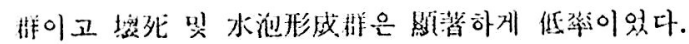

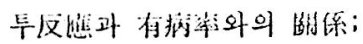

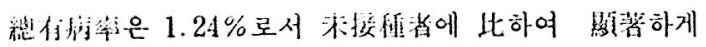

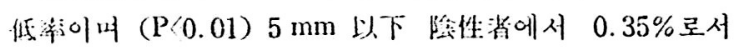

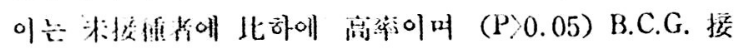

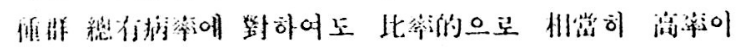
있다.

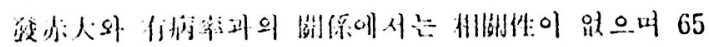

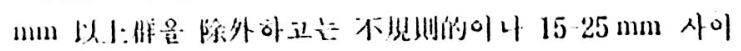

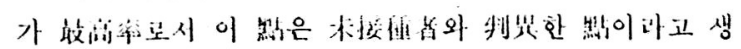
각된다.

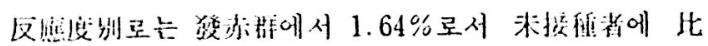

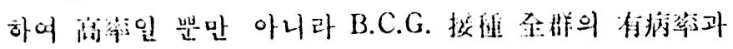

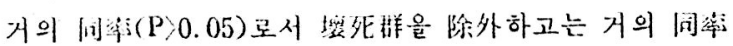
이있다 (8-a.b).

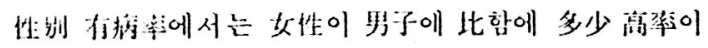

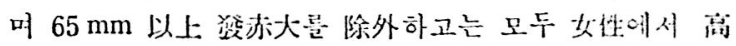
率이었다 (P>0.05) (9-a.b).

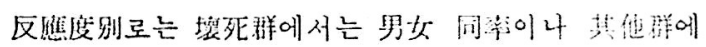

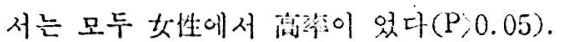

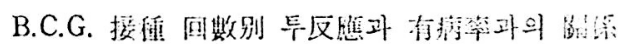

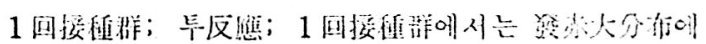

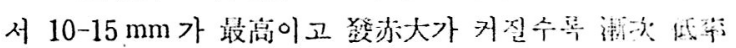
이었다.

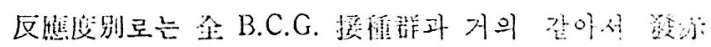
群이 最话的있다.

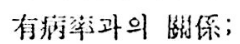

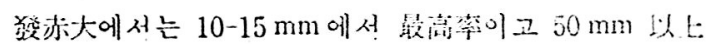

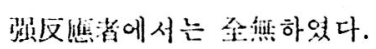

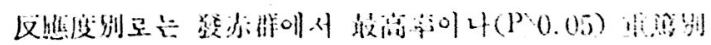

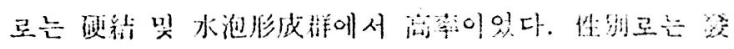

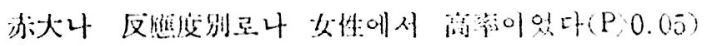
$(10 a, b, 102 a, b)$.

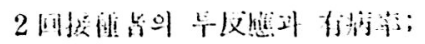

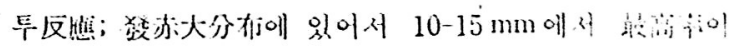
고 다음이 15-20 mm이며 $30 \mathrm{~mm}$ 以上에서 1 잢 比하여 低然이있다.

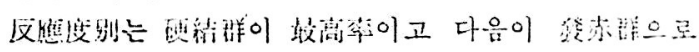

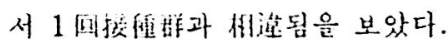

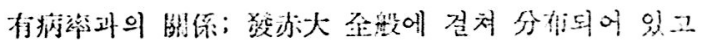


Vol. 15, No. 4, Cctoter, 1268

Tcble 102 a. Correlation between radiolngical findings and tuberculin reaction in vaccinatees by sex (once).

\begin{tabular}{|c|c|c|c|c|c|c|c|c|c|c|c|c|c|c|c|}
\hline Anic & Size & 5 & $10^{\prime}$ & 15 & 20 & 25 & 30 & 35 & 40 & 15 & 50 & 55 & 60 & 65 & Total \\
\hline Tuberculis: & No. & 565 & 75 & 95 & 85 & 65 & 53 & 34 & 23 & 26 & 26 & 19 & 21 & 41 & 1131 \\
\hline $\begin{array}{l}\text { Renctin! } \\
\text { in S:ze }\end{array}$ & $\mathrm{M}^{2}$ & $\begin{array}{r}49 . \\
96 \\
2\end{array}$ & 6.63 & $\begin{array}{r}8.10 \\
3\end{array}$ & $\begin{array}{r}7.52 \\
1\end{array}$ & $\begin{array}{r}5.75 \\
1\end{array}$ & $\begin{array}{r}4.69 \\
1\end{array}$ & $\begin{array}{r}3.01 \\
1\end{array}$ & 2.03 & 2.29 & 2.29 & 1.18 & 2.12 & 3.63 & \\
\hline Raciona! & $M$ & 1 & & 1 & 1 & & & 1 & & & & & & & \\
\hline Sunet: & $T+a !$ & 3 & & 4 & 2 & 1 & 1 & 1 & & & & & & & 12 \\
\hline & $\because:$ & 0.58 & & 4.21 & 2.35 & 1.54 & 1.89 & 2.94 & & & & & & & 1.0 \\
\hline cmals & $5 i a c$ & 5 & 10 & 15 & 20 & 25 & $80^{\prime}$ & 35 & 40 & 45 & 50 & 5.5 & 60 & $6: 1$ & Tolat \\
\hline Tutercelin & $\therefore$. & 457 & 86 & 94 & 68 & 58 & 31 & 22 & 23 & 25 & 25 & 22 & 26 & 37 & 97 \\
\hline $\begin{array}{l}\text { Kenction in } \\
\text { Size }\end{array}$ & s: & $\begin{array}{r}47 . \\
80\end{array}$ & 8.80 & 9.62 & 6.96 & 1.94 & 3.18 & 2.25 & 2.35 & 2.56 & 2.56 & 2.25 & 2.66 & 3.79 & \\
\hline & $M$ & & 2 & 4 & 1 & 1 & & & & & & & & & \\
\hline Reciogal & $M$ & & & & 1 & 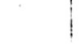 & & 1 & & & 1 & & & & \\
\hline Suscots & $T+\mathrm{C}$ & 4 & 2 & "it & 2 & 1 & & 1] & & & 1 & & & & 13 \\
\hline & $\therefore$ & 0.14 & 2.33 & 4.26 & 2.94 & 1. 72 & & 4.55 & & & 4. Co & & & & 1.23 \\
\hline
\end{tabular}

Toble 1 - 2 b. Correlation between rediological findings and tuberculin reaction in vaccinatecs by sex (once).

\begin{tabular}{|c|c|c|c|c|c|c|c|}
\hline & Intensity & Erythema & $\begin{array}{l}\text { Double } \\
\text { Erythoma }\end{array}$ & Induration & Necrosis & Blcbs & Total \\
\hline $\begin{array}{l}\text { Male } \\
\text { Intensity } \\
\text { Tuberculin } \\
\text { Reation } \\
\text { Radiological } \\
\text { Suspects }\end{array}$ & $\begin{array}{l}\text { No. } \\
\% \\
M \\
\text { MiA } \\
\text { Total } \\
\%\end{array}$ & $\begin{array}{r}240 \\
41.67 \\
4 \\
1 \\
5 \\
2.08\end{array}$ & $\begin{array}{r}68 \\
11.81\end{array}$ & $\begin{array}{r}213 \\
36.98 \\
3 \\
1 \\
4 \\
1.88\end{array}$ & $\begin{array}{r}23 \\
3.99\end{array}$ & $\begin{array}{r}32 \\
5.56\end{array}$ & 586 \\
\hline $\begin{array}{l}\text { Fomale } \\
\text { Intonsity } \\
\text { Tubracuin } \\
\text { Reaction } \\
\text { Rediongi al } \\
\text { Suspects }\end{array}$ & $\begin{array}{c}\text { No. } \\
\text { Có } \\
\text { Mi } \\
\text { MA } \\
\text { Trial } \\
\text { 的 }\end{array}$ & $\begin{array}{r}217 \\
41.73 \\
5 \\
5 \\
5 \\
2.30\end{array}$ & $\begin{array}{r}82 \\
15.77 \\
1 \\
\\
1 \\
1.22\end{array}$ & $\begin{array}{r}172 \\
33.08 \\
2 \\
2 \\
4 \\
2.33\end{array}$ & $\begin{array}{r}21 \\
4.62\end{array}$ & $\begin{array}{r}25 \\
4.81 \\
1 \\
1 \\
4.00\end{array}$ & 520 \\
\hline
\end{tabular}

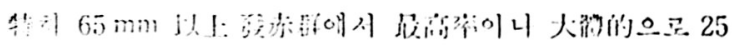

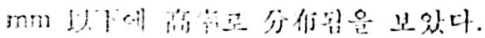

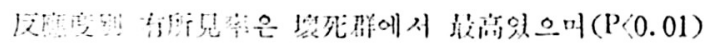

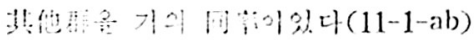

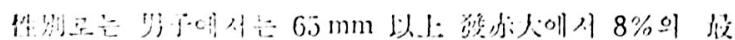

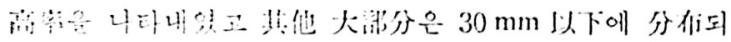

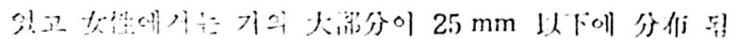

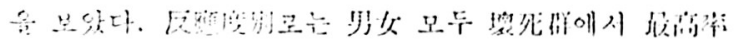

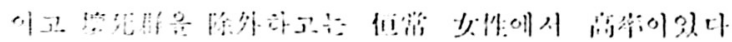
(P.0.06).

3 S.C.C. B.

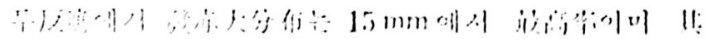

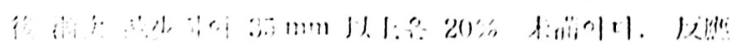

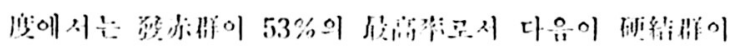

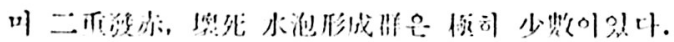

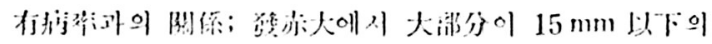

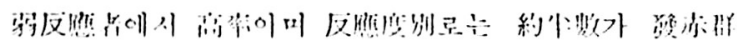

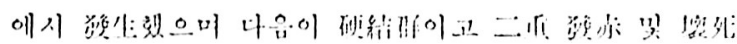

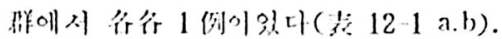

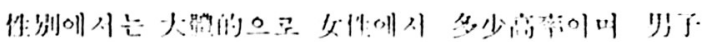

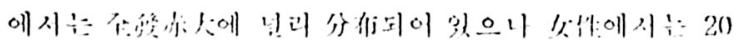

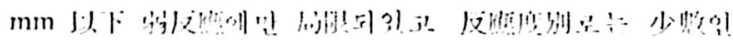

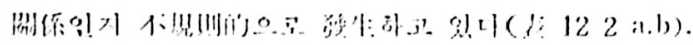

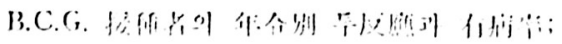

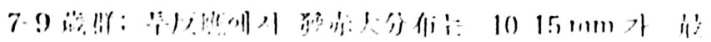

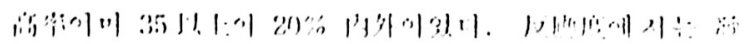




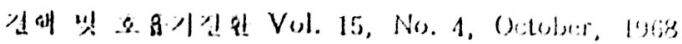

Table 111 a. Correlation between radiological fiindings and tuberculin reaction in vaccinateses ('l'wice).

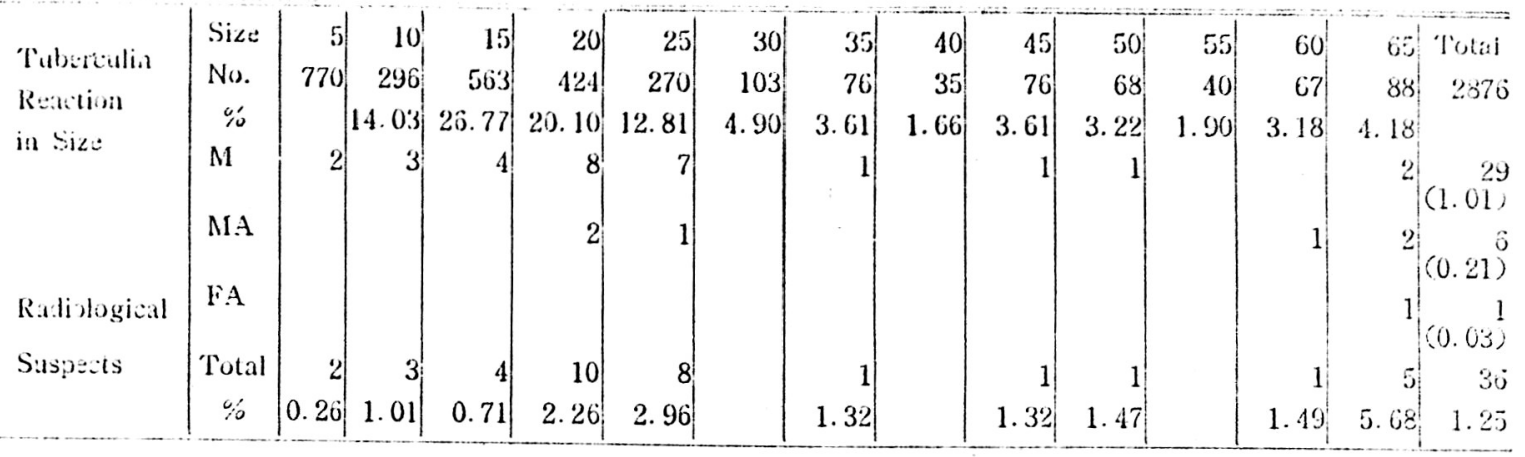

Table 11-1-b. Correlation between radiological fiindings and tuberculin reaction in vaccinatees (Twice).

\begin{tabular}{l|c|r|r|r|r|r|r} 
& Intensity & Erythema & $\begin{array}{c}\text { Double } \\
\text { Erythema }\end{array}$ & Induration & Necrosis & Blebs & Total \\
\hline & No. & 791 & 326 & 857 & 82 & 50 & 2106 \\
Intensity & $\%$ & 37.60 & 15.45 & 40.70 & 3.89 & 2.37 & \\
Tubsrculin & M & 9 & 4 & 13 & 1 & & 27 \\
Reaction & MA & 1 & 1 & 1 & 3 & & 6 \\
& FA & & & & 1 & & 1 \\
& Total & 10 & 5 & 14 & 5 & & 34 \\
& M & 1.14 & 1.23 & 1.52 & 1.22 & & 1.28 \\
Radiological & MA & 0.13 & 0.30 & 0.11 & 3.15 & & 0.28 \\
Suspects & FA & & & & 1.18 & & 0.05 \\
& Total & 1.27 & 1.53 & 1.63 & 5.55 & & 1.61 \\
\hline
\end{tabular}

Table 11-2-a Correlation between radiological findings and tuberculin reaction in vaccinatees by sex. ('Twice).

\begin{tabular}{|c|c|c|c|c|c|c|c|c|c|c|c|c|c|c|c|}
\hline Male & Size & 5 & $10^{\prime}$ & 15 & 20 & $2 \overline{5}$ & $30^{\prime}$ & 35 & 40 & 45 & 50 & 55 & $60^{\prime}$ & 65 & Total \\
\hline Tuberculin & No. & 475 & 184 & 352 & 260 & 182 & 71 & 48 & 15 & 39 & 36 & 23 & 30 & 47 & 1768 \\
\hline Reaction in & $\%$ & & 14.22 & 27.20 & 20.10 & 14.10 & 5.48 & 3.72 & 1.16 & 3.01 & 2.78 & 1.78 & 2.78 & 3.63 & \\
\hline Size & $M$ & 1 & & & & & 1 . & & & & & & & & $\begin{array}{r}14 \\
(0.79)^{2}\end{array}$ \\
\hline & $\mathrm{MA}$ & & & 1 & 1 & & & & & 0 & & & 1 & & \\
\hline Radiological & FA & & & & & & & & & & & & & & 1 \\
\hline Suspects & Total & 1 & & 5 & 4 & & 1 & & & & & & & 4 & 19 \\
\hline & $\%$ & $|0.54|$ & 0.83 & 1.92 & 2.50 & & 2.08 & & & & & & 1.78 & 8.52 & 1.07 \\
\hline Female & Size & 5 & 10 & 15 & 20 & 25 & 30 & 35 & 40 & 45 & 50 & 55 & 60 & 65 & Total \\
\hline Tuberculin & No. & 295 & 112 & 211 & 164 & 88 & 32 & 28 & 20 & 37 & 32 & 17 & 31 & 41 & 1103 \\
\hline Reaction in & $\%$ & & $13.80^{\prime}$ & 20.00 & 20.20 & 18. 80 & 3.93 & 3.44 & 2.40 & 4.55 & 3.93 & 2.09 & 3.82 & 5.05 & \\
\hline Size & $M$ & 2 & & & & 4 & & & & 1 & & & & & 15 \\
\hline & $\mathrm{MA}$ & & & & 1 & & & & & & & & & 1 & 2 \\
\hline Radiological & Total & 2 & 2 & 1 & ju & 4 & & & & 1 & 1 & & & 2 & 17 \\
\hline Sispects & $\%$ & 0.68 & 1.78 & 0.47 & 3.01 & 4.55 & & & & 2.70 & 3.13 & & & $2.44_{i}$ & 1.35 \\
\hline
\end{tabular}




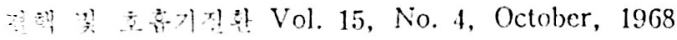

Table 11-2-b. Correlation between radiological fiindings and tuberculin reaction in raccinates by sex. (Twice).

\begin{tabular}{|c|c|c|c|c|c|c|c|}
\hline & Intensity & Erythema & $\begin{array}{l}\text { D.uhble } \\
\text { Erythema }\end{array}$ & Incluratien & Nacrosis & Blebs & Tontal \\
\hline \multirow{7}{*}{$\begin{array}{l}\text { Male } \\
\text { Tuberculin } \\
\text { Reaction in } \\
\text { Size }\end{array}$} & No. & 471 & 233 & 513 & 54 & 22 & 2100 \\
\hline & $\%$ & 36.10 & 18.00 & 39.70 & 4.17 & 1.70 & \\
\hline & $M$ & 4 & 2 & 7 & 1 & & 14 \\
\hline & $M A$ & 1 & 1 & & 2 & & 4 \\
\hline & FA & & & & 1 & 1 & 1 \\
\hline & Total & 5 & 3 & 7 & 4 & & 19 \\
\hline & $\mathrm{M}$ & 0.85 & 0.83 & 1.36 & 1.85 & & 0.67 \\
\hline \multirow{3}{*}{$\begin{array}{l}\text { Radiological } \\
\text { Suspects }\end{array}$} & $M A$ & 0.21 & 0.42 & & 3.70 & & 0.19 \\
\hline & FA & & & & 1.85 & & 0.05 \\
\hline & Total & 1.06 & 1.28 & 1.26 & 7.40 & & 0.91 \\
\hline \multirow{5}{*}{$\begin{array}{l}\text { Female, } \\
\text { Tubcreulin } \\
\text { Reaction in } \\
\text { Sizc }\end{array}$} & No. & 320 & 93 & 314 & 23 & 28 & 1293 \\
\hline & $\%$ & 39.40 & 11.42 & 12.40 & 3.45 & 3.45 & \\
\hline & $M$ & 5 & 2 & 6 & & & 13 \\
\hline & $M A$ & & & 1 & 1 & & 2 \\
\hline & Total & 5 & 2 & 7 & 1 & & 15 \\
\hline \multirow{3}{*}{ Radiogical } & M & 1.56 & 2.16 & 1.74 & 3.58 & & 1.01 \\
\hline & MA & & & 0.29 & & & 0.16 \\
\hline & Total & 1.56 & 2.16 & 2.03 & 3.58 & & 1.17 \\
\hline
\end{tabular}

Table 12-1. Correlation between radiological fiindings and tuberculin reaction in vaccinatces (thrice).

\begin{tabular}{|c|c|c|c|c|c|c|c|c|c|c|c|c|c|c|c|}
\hline Tuberculin & Sizc & 5 & 10 & 15 & 20 & 25 & 30 & 35 & 40 & 45 & 50 & 55 & 60 & 65 & Total \\
\hline Reaction in & No. & 192 & 86 & 185 & 141 & 62 & 33 & 28 & 19 & 21 & 18 & 12 & 10 & 10 & 822 \\
\hline & $\%$ & & 13.64 & 29.40 & 22.10 & 9.85 & 5.24 & 4.45 & 3.02 & 3.14 & 2.86 & 1.91 & 2.38 & 1.59 & \\
\hline Radiological & $\begin{array}{l}\mathrm{M} \\
\mathrm{MA}\end{array}$ & 1 & $\begin{array}{l}5 \\
1\end{array}$ & 2 & & & & & & 1 & & & & 4 & $\begin{array}{r}1.09) \\
2 \\
0.30)^{2}\end{array}$ \\
\hline Suspects & $\begin{array}{c}\text { Total } \\
\%\end{array}$ & $\begin{array}{r}1 \\
1.16\end{array}$ & $\begin{array}{r}6 \\
3.24\end{array}$ & $\begin{array}{r}2 \\
1.42\end{array}$ & & & & & & $\begin{array}{r}1 \\
4.77\end{array}$ & & & & $\begin{array}{r}1 \\
10.00\end{array}$ & $\begin{array}{r}11 \\
1.39\end{array}$ \\
\hline
\end{tabular}

\begin{tabular}{|c|c|c|c|c|c|c|c|}
\hline & Intensity & Erythema & $\begin{array}{l}\text { Do:the } \\
\text { Erythema }\end{array}$ & Induration & Necrosis & Blebs & lotal \\
\hline Tuberculin & No. & 331 & 99 & 163 & 20 & 14 & 630 \\
\hline Reaction & $\%$ & 53.15 & 15.70 & 25.85 & 3.15 & 2.20 & \\
\hline in $\operatorname{Sizc}$ & $M$ & 5 & 1 & 3 & 0 & & $(1.42)^{9}$ \\
\hline & $\mathrm{MA}$ & 1 & & & 1 & & $(0.32)^{2}$ \\
\hline Rardinelerical & Tot:al & 6 & 1 & 3 & 1 & & 11 \\
\hline Suepects & $\%$ & 1. 79 & 1.01 & 1.81 & 5.00 & & 1.74 \\
\hline
\end{tabular}


Table 122 a. Correlation between radiological fiindings and tuberculin reaction vatcinatees by sex(thrice).

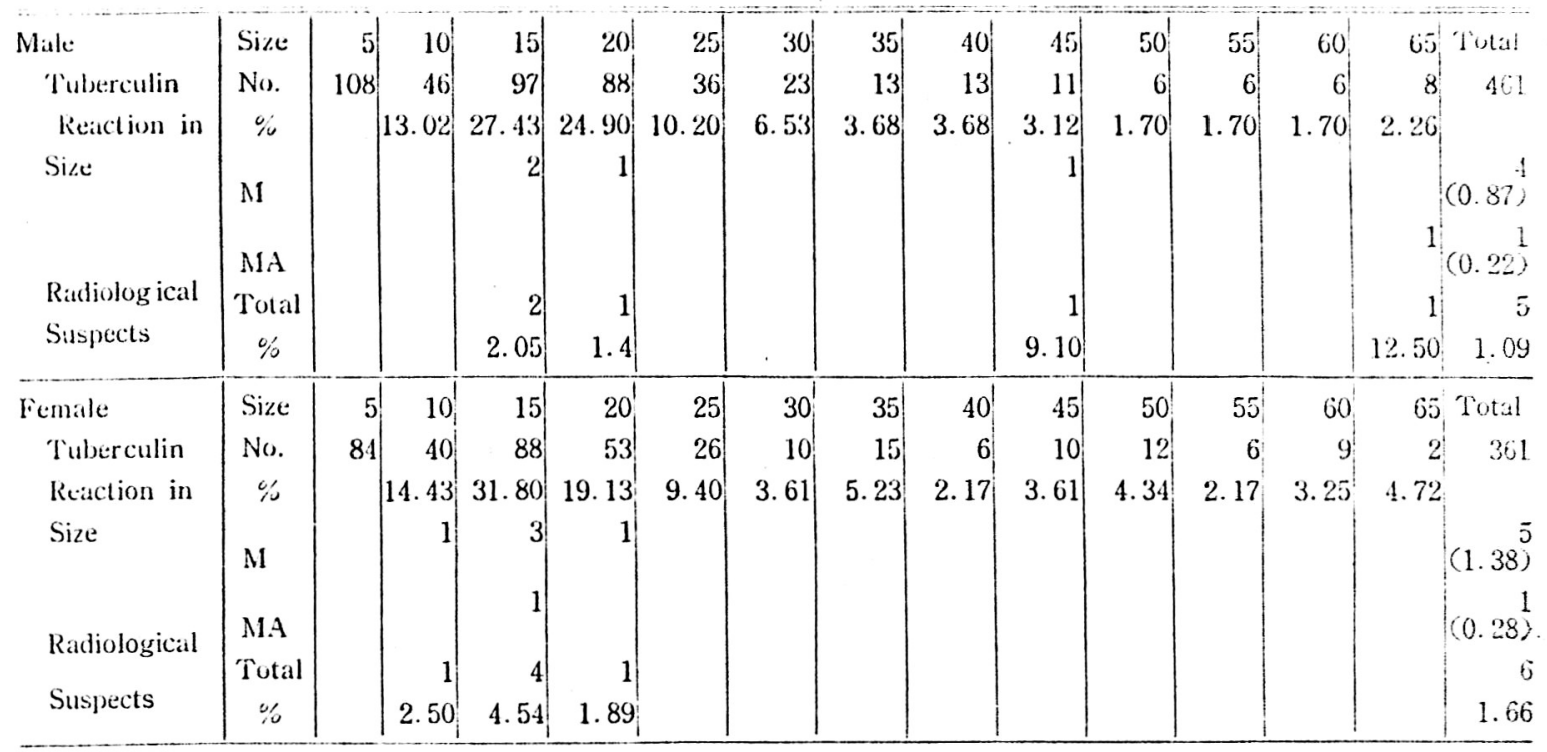

Table $12-2$ b. Correlation between radiological findings and tuberculin reaction vaccinatees by sex(thrice).

\begin{tabular}{|c|c|c|c|c|c|c|}
\hline & Intensity & Erythema & $\begin{array}{l}\text { Double } \\
\text { Erythema }\end{array}$ & Induration & Necrosis & Bleds \\
\hline \multirow{4}{*}{$\begin{array}{l}\text { Male } \\
\text { Tubercu } \\
\text { Ruiction } \\
\text { in Size }\end{array}$} & No. & 200 & 46 & 91 & 10 & 6 \\
\hline & $\%$ & 56.65 & 13.00 & 25.35 & 2.85 & 1.71 \\
\hline & $M$ & 3 & & 1 & & \\
\hline & MA & & & & 1 & \\
\hline \multirow{2}{*}{$\begin{array}{l}\text { Radiological } \\
\text { Suspects }\end{array}$} & Total & 3 & & 1 & 1 & \\
\hline & $\%$ & 1.50 & & 1.10 & 10.00 & \\
\hline \multirow{5}{*}{$\begin{array}{l}\text { Female } \\
\text { Tuberculin Reaction } \\
\text { in Size }\end{array}$} & No. & 134 & 53 & 72 & 10 & 8 \\
\hline & $\%$ & 48.50 & 19.15 & 26.00 & 3.61 & 2.89 \\
\hline & M & 2 & 1 & 2 & & \\
\hline & $\mathrm{MA}$ & 1 & & & & \\
\hline & Total & 3 & 1 & 2 & & \\
\hline Radiological Suspects & $\%$ & 2.24 & 1.89 & 2.78 & & \\
\hline
\end{tabular}

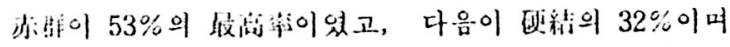

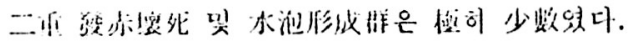

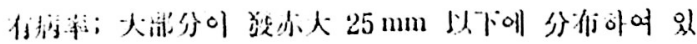

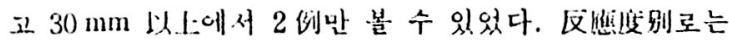

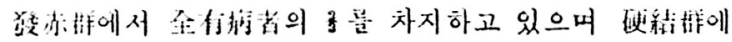

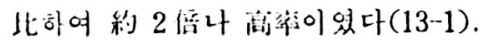

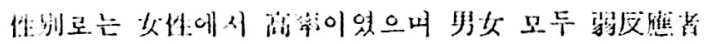

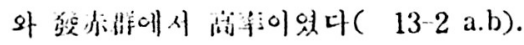

10-13 获据：

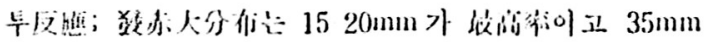

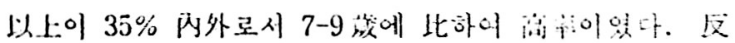

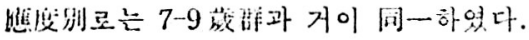

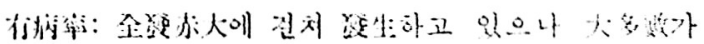

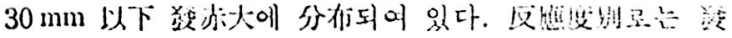

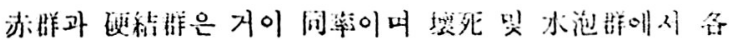
各 1 例卅 볼 全 있었다(洯 14).

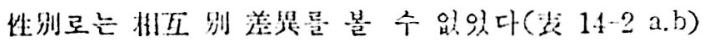
14-16 植群；

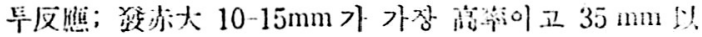

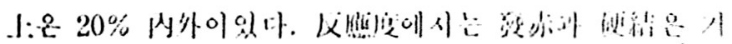


견 1? Vol. 15. No. 4, October, 1968

Table $131 \mathrm{a} . \quad$ Correlation betwecn radiologrical findings and tubcrculin reaction in vaccinatees by age group 7-9

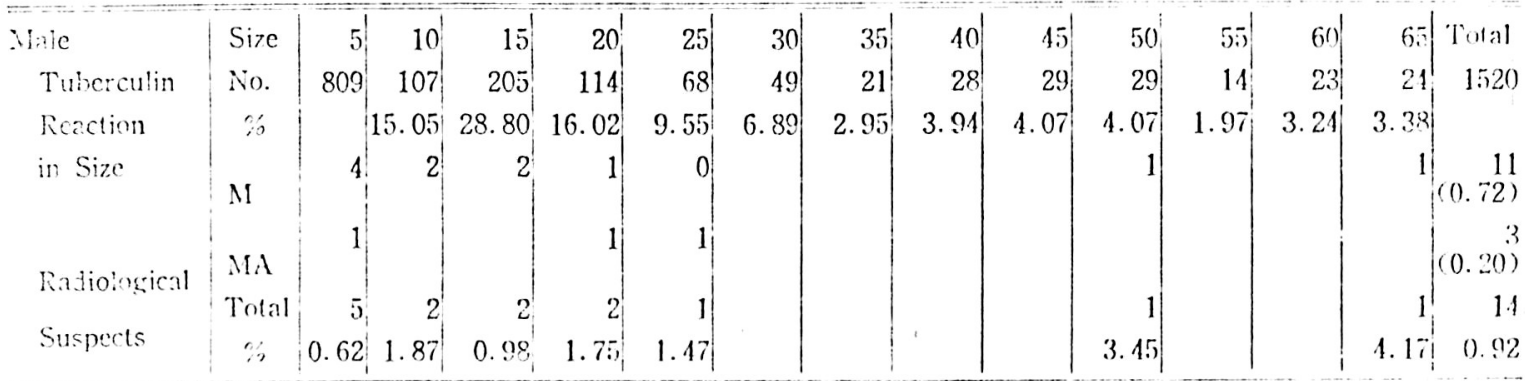

Table $131 \mathrm{~b}$. Correlation ation between radiolngical findings and tuberculin reaction in vaccinatecs by age group 79

\begin{tabular}{|c|c|c|c|c|c|c|c|}
\hline & Intensity & Erythema & $\begin{array}{l}\text { Double } \\
\text { Erythema }\end{array}$ & Induration & Necrosis & Blebs & Tintal \\
\hline Fomale & No. & $3 \pi$ & 51 & 232 & 16 & 31 & 311 \\
\hline Tuberculin & $\%$ & 53.00 & 7.17 & 32.71 & 2.25 & 4.92 & \\
\hline Recetion in & M & 6 & & 1 & & & $(0.99)^{7}$ \\
\hline $5: 20$ & $M A$ & & & 1 & 1 & & $\begin{array}{r}2 \\
(0.28)^{2}\end{array}$ \\
\hline Radiolosical & Total & & & 6 & 2 & & 9 \\
\hline Suspects & $\%$ & 1.59 & & 0.86 & 6.25 & & 1.27 \\
\hline
\end{tabular}

Table 13-2-a. Correlation between radiological findings and tuberculin reaction in

vaccinatees by age group 7-9

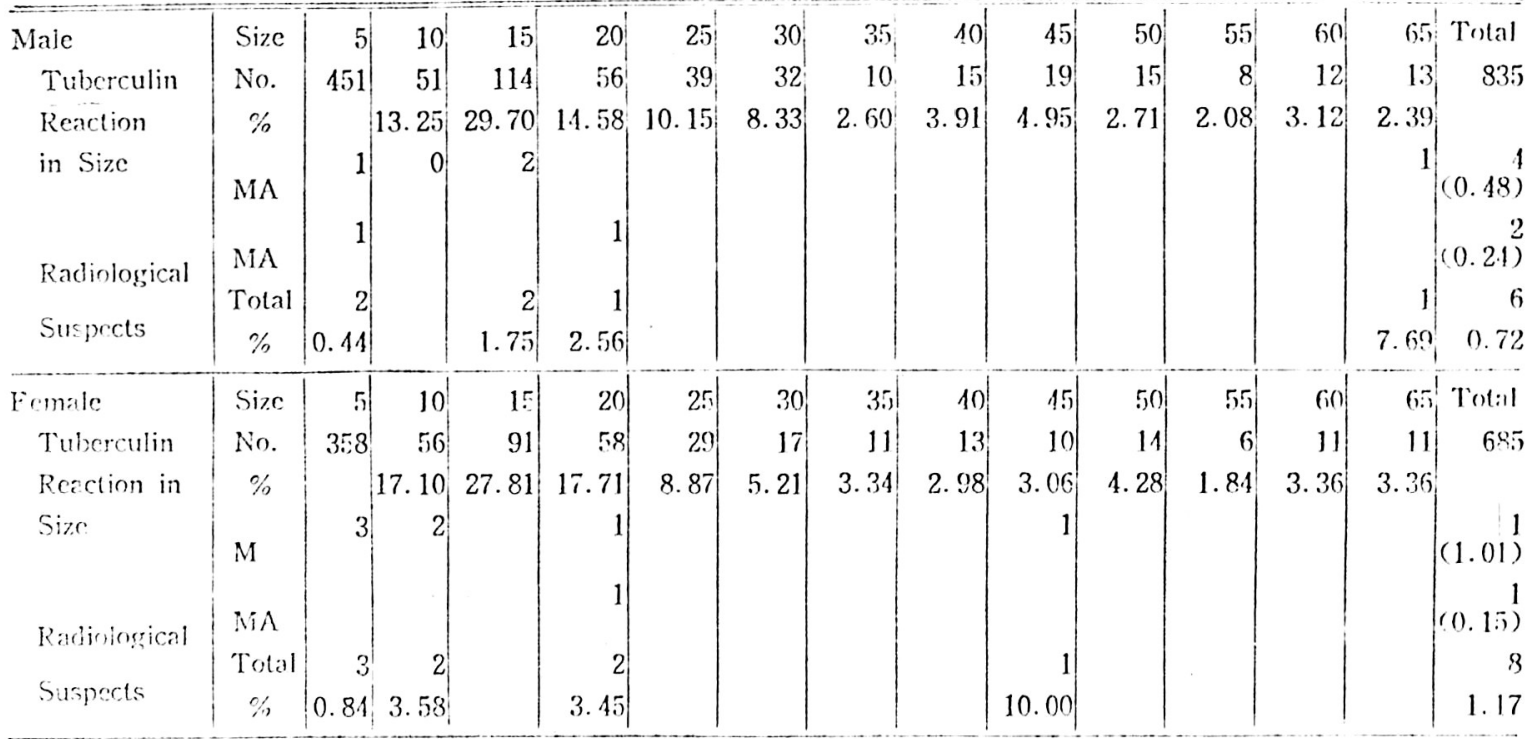

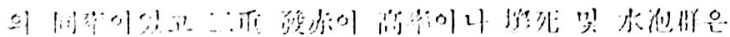

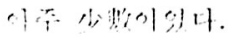

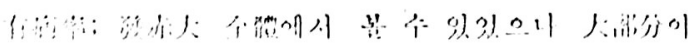
a)

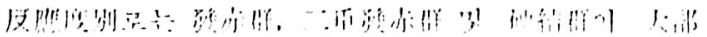

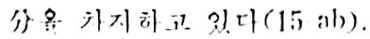

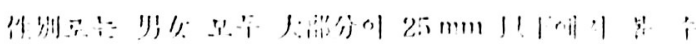

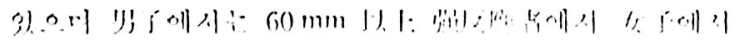




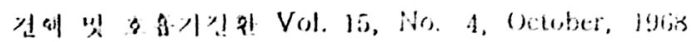

Tabl = $132 \mathrm{~b}$. Correlation between radiological findings and tuberculin reaction in vaccinatees by age group $7-9$

\begin{tabular}{|c|c|c|c|c|c|c|c|}
\hline & Intensity & Erythema & $\begin{array}{l}\text { Double } \\
\text { Erytheina }\end{array}$ & Induration & Necrosis & Elets & Tuli:! \\
\hline Male & No. & 190 & 23 & 136 & 12 & 23 & 384 \\
\hline 'I'uberculin & $\%$ & 49.49 & 6.01 & 35.43 & 3.12 & 0.00 & \\
\hline Reaction in & $\mathrm{M}$ & 3 & & & & & $\left(0.73^{3}\right.$ \\
\hline Size & MA & & & & 1 & & 1 \\
\hline Radiological & Total & 3 & & & 1 & & t \\
\hline Suspects & $\%$ & 1.58 & & & 6.34 & & 1.0 .4 \\
\hline Female & No. & 187 & 28 & 93 & 4 & 12 & 327 \\
\hline Tuberculin & $\%$ & 57.19 & 8.56 & 29.28 & 1.23 & $3.5 j$ & \\
\hline Reaction & $\mathrm{M}$ & 3 & & 1 & & & \\
\hline in Size & $\mathrm{MA}$ & & & 1 & & & $\begin{array}{r}(1.23) \\
1\end{array}$ \\
\hline Radiological & & & & & & & $(0.31)$ \\
\hline Suspects & $\begin{array}{c}\text { Total } \\
\%\end{array}$ & $\begin{array}{r}3 \\
1.60\end{array}$ & & $\begin{array}{r}2 \\
2.08\end{array}$ & & & $\begin{array}{r}5 \\
1.54\end{array}$ \\
\hline
\end{tabular}

Table 14. 1 a Correlation between radiological and tuberculin reaction in vaccinatees by age group 10-13

\begin{tabular}{l|c|r|r|r|r|r|r|r|r|r|r|r|r|r|r|r|r}
\hline Tuberculin & Size & 5 & 10 & 15 & 20 & 25 & 30 & 35 & 40 & 45 & 50 & 55 & 60 & 65 & Total \\
Reaction & No. & 565 & 116 & 221 & 248 & 143 & 79 & 49 & 38 & 59 & 61 & 46 & 52 & 54 & 1731 \\
in Size & $\%$ & & 9.95 & 18.09 & 21.25 & 12.28 & 6.78 & 9.21 & 3.26 & 5.06 & 5.23 & 3.95 & 4.46 & 4.63 & \\
& $\mathrm{M}$ & 1 & & 3 & 5 & 2 & 1 & & & 1 & & & & & 13 \\
& & & & & 1 & & & & & & & & & 1 & 1 \\
Radiological & MA & & & & & & & & & & 1 & & & & $(0.17)$ \\
Suspects & Total & 1 & & 3 & 6 & 2 & 1 & & & 1 & 1 & & & 1 & 16 \\
\hline
\end{tabular}

Table $141 \mathrm{~b}$. Correlation between radiological findings and tuberculin reaction in vaccinalees by age group $10-13$

\begin{tabular}{|c|c|c|c|c|c|c|}
\hline & Intensity & Erythema & $\begin{array}{l}\text { Double } \\
\text { Erythema }\end{array}$ & Induration & Necrosis & Blebs \\
\hline l'uberculin & No. & 602 & 87 & 385 & 24 & is \\
\hline Reaction in & $\%$ & 51.60 & 7.46 & 33.09 & 2.04 & 5.33 \\
\hline \multirow[t]{2}{*}{ Size } & M & 6 & & 6 & & \\
\hline & $\mathrm{MA}$ & 1 & & & 1 & 1 \\
\hline Radiolo sical & Total & 7 & & 6 & 1 & 1 \\
\hline Suspects & $\%$ & 1.16 & & 1.50 & 4.17 & 1.49 \\
\hline
\end{tabular}

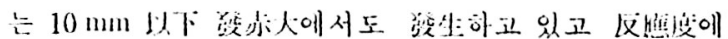

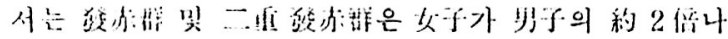

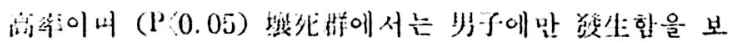
았다( 152 a.b).
考察

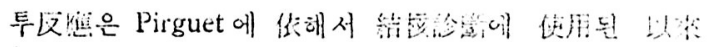

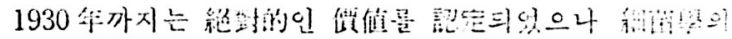

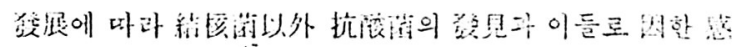


\%

Table 14-2-a. Corrclation between radiological and tul;crculin reaction in vaccinatees by age group 10-13

\begin{tabular}{|c|c|c|c|c|c|c|c|c|c|c|c|c|c|c|c|}
\hline Male & sizc & 5 & 10 & 15 & 20 & 25 & 30 & 35 & 40 & 45 & 50 & 55 & 60 & $65^{\prime}$ & Total \\
\hline Tuberculin & No. & 296 & 58 & 127 & 1.57 & 86 & 43 & 24 & 22 & 25 & 30 & 26 & 21 & 29 & 914 \\
\hline Raction in & $\therefore$ & & 8.95 & 19.60 & 21.21 & 13.28 & 6.65 & 3.70 & 3.40 & 3.86 & 4.64 & 4.02 & 3.24 & 4. 48 & \\
\hline Size & $M$ & 1 & & & & & & & & 1 & & & & & $\begin{array}{r}7 \\
(0.74)^{7}\end{array}$ \\
\hline Barolorical & $M ! A$ & & & & 1 & & & & & & & & & & $(0.11)^{1}$ \\
\hline & Total & 1 & & 1 & 4 & 1 & & & & 1 & & & & & \\
\hline Suspeits & $\therefore$ & 0.34 & & 0.79 & 2.55 & 2.33 & & & & 4.00 & & & & & 0.85 \\
\hline Female & $\operatorname{sinc}$ & 5 & 10 & 15 & 20 & 25 & 30 & 35 & 40 & 45 & 50 & 55 & $60^{\circ}$ & (65) & Total \\
\hline Tuberculin & No. & 269 & 58 & 94 & 91 & 57 & 36 & 25 & 16 & 31 & 31 & 20 & 31 & 25 & $78 \%$ \\
\hline Reaction in & $\%$ & & 11.18 & 18.12 & 17.58 & 11.00 & 6.95 & 4.83 & 3.09 & 6.56 & 5.98 & 3. 86 & 5.98 & 4.83 & \\
\hline Size & $M$ & & & & & & & & & & & & & & $\begin{array}{r}6 \\
(0.75)^{6}\end{array}$ \\
\hline Radiolnovical & M.t & & & & & & & & & & 1 & & & & $(0.12)^{1}$ \\
\hline & Total & & & 2 & 2 & 2 & & & & & 1 & & & & 7 \\
\hline Suspets & $\%$ & & & 2.13 & 2.20 & 3.52 & & & & & 3.23 & & & & 0.87 \\
\hline
\end{tabular}

Toble 14-2-b. Correlation between radiological and tubcrculin reaction in vaccinatces by age group 10-13

\begin{tabular}{|c|c|c|c|c|c|c|}
\hline & Intensity & Erythema & $\begin{array}{l}\text { Double } \\
\text { Erythema }\end{array}$ & Induration & Necrosis & Blcbs \\
\hline Male & No, & 334 & 46 & 226 & 11 & 31 \\
\hline Tuberculin & $\%$ & 51.60 & 7.11 & 34.75 & 1.70 & 4.78 \\
\hline Reaction in & $\mathrm{M}$ & 3 & & 3 & & \\
\hline Size & MA & 1 & & & 1 & \\
\hline Radiolngical & Total & 4 & & 3 & 1 & \\
\hline Suspects & $\%$ & 1.21 & & 1.32 & 9.11 & \\
\hline Femalc & No. & 268 & 41 & 159 & 13 & 37 \\
\hline Tubcrculin & $\%$ & 51.70 & 7.91 & 30.73 & 2.51 & 7.15 \\
\hline Reacten in & M & 3 & & 3 & & \\
\hline Sizc & MA & & & & & 1 \\
\hline Radiolngical & Total & 3 & & 3 & & 1 \\
\hline Suspects & $\%$ & 1.12 & & 1.89 & & 2.70 \\
\hline
\end{tabular}

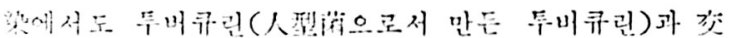

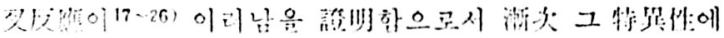

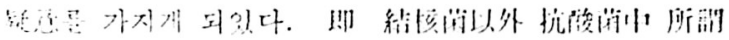

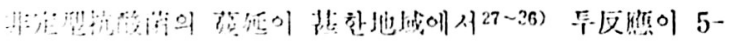

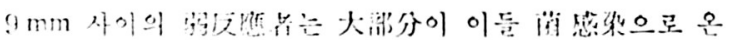

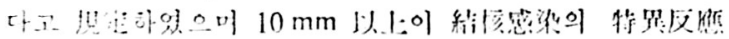

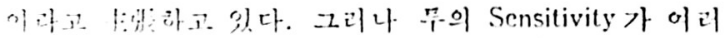

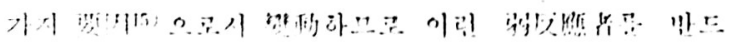

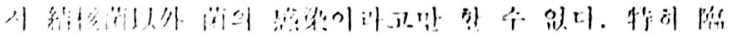

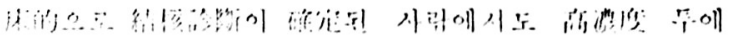

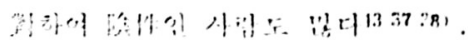

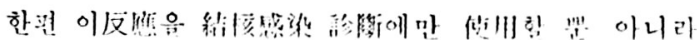

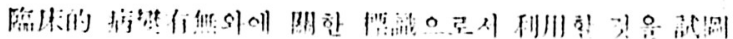

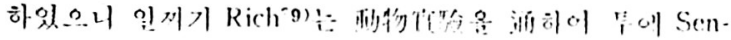

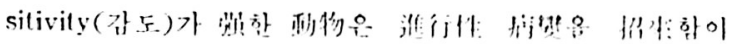

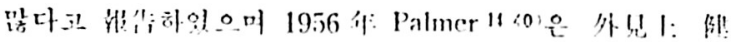

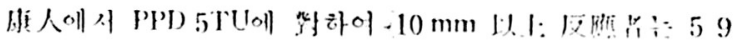

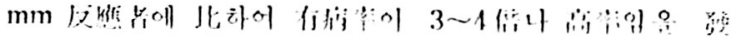

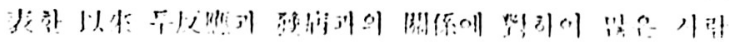

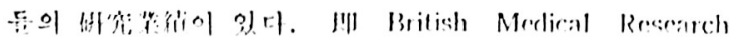

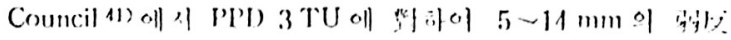

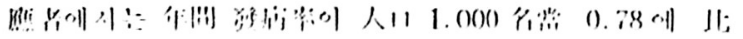




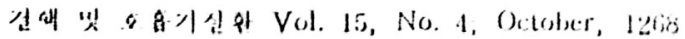

Toble 152 A. Correlation between radiological findings and tuberculin reaction in vaccinatees by ange group 1416

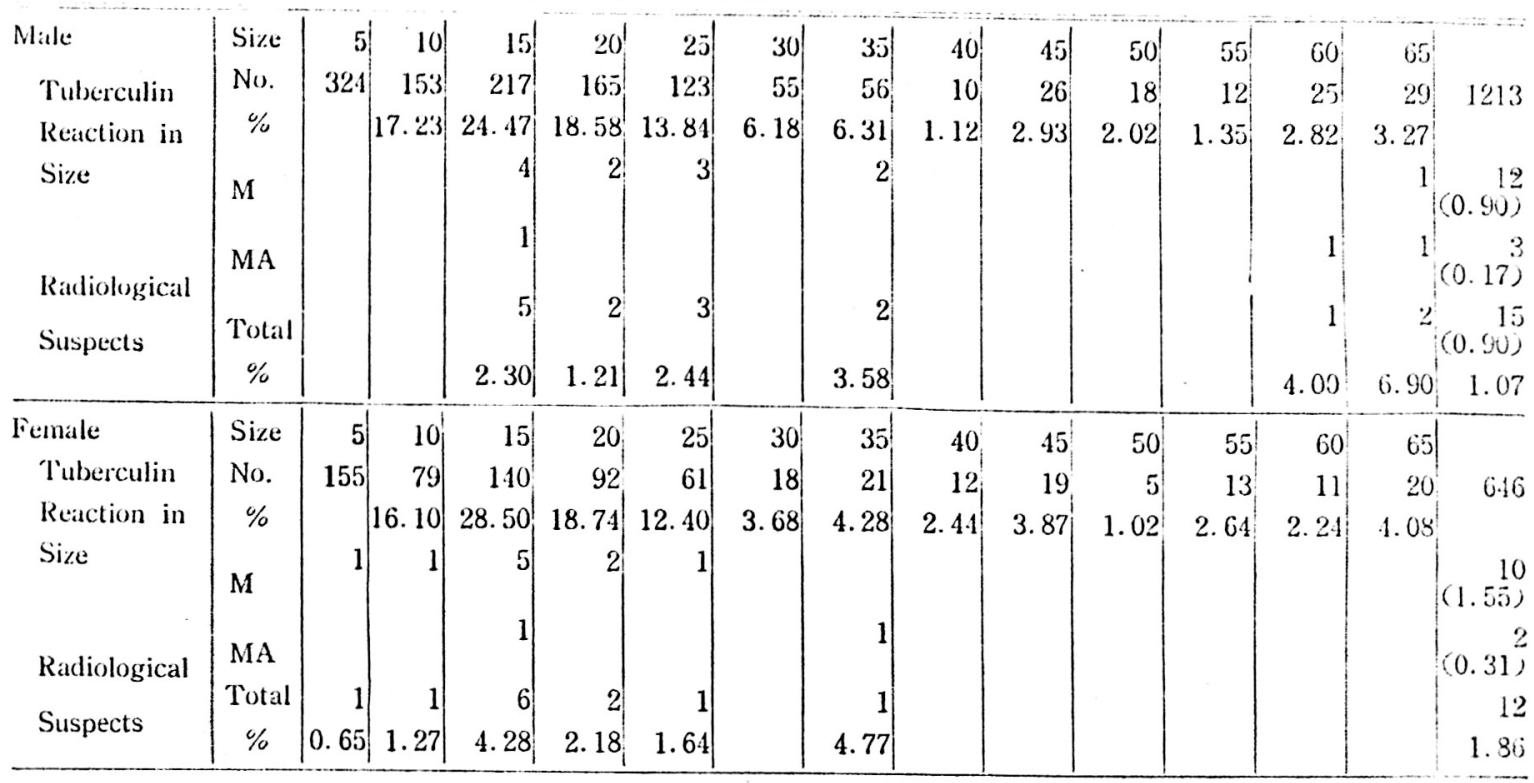

Table 15 A. Correlation between radiological fiindings and tuberculin reaction in vaccinatees by age group 14-16

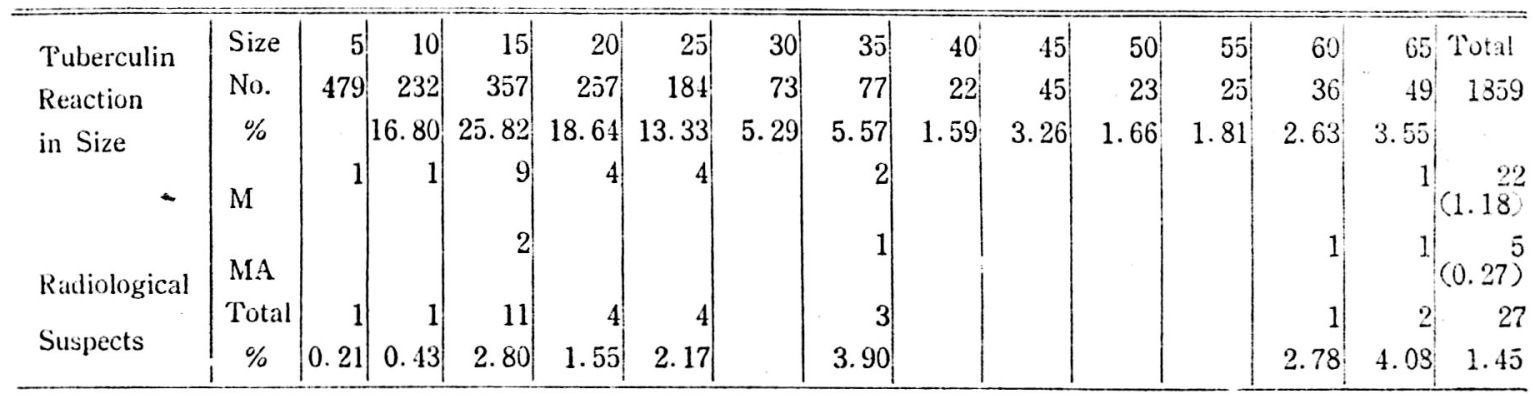

Table 15-B. Correlation between radiological fiindings and tuberculin reaction in vaccinatees by age group 14-16

\begin{tabular}{l|c|r|r|r|r|r}
\hline & Intensity & Erythema & $\begin{array}{c}\text { Double } \\
\text { Erythema }\end{array}$ & Induration & Necrosis & Blebs \\
\hline Intensity & No. & 485 & 367 & 446 & 67 & 15 \\
Tuberculin Reaction & $\%$ & 35.15 & 26.60 & 32.35 & 4.85 & 1.09 \\
& $\mathrm{M}$ & 7 & 5 & 8 & 1 & \\
& $\mathrm{MA}$ & 1 & 1 & 2 & 1 & 2 \\
Radiological & Total & 8 & 6 & 10 & 1.49 & \\
Suspects & $\mathrm{M}$ & 1.44 & 1.36 & 1.79 & 1.50 & \\
& $\mathrm{MA}$ & 0.16 & 0.27 & 0.55 & 2.99 & \\
\hline
\end{tabular}

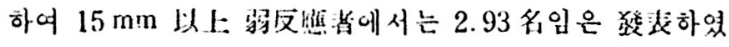

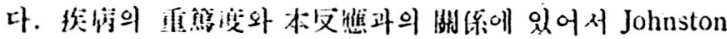

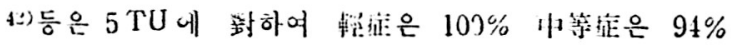

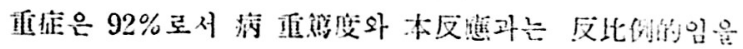

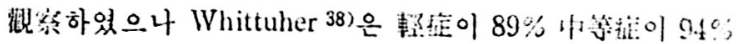

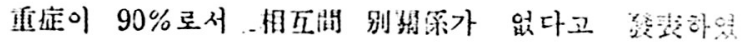




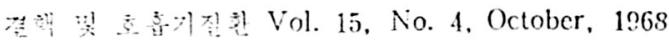

Table 15-2-B. Correaltion between radiological findings and tuberculin reaction in raccinatces by age group $11-16$

\begin{tabular}{|c|c|c|c|c|c|c|}
\hline & Intensity & Erythema & $\begin{array}{l}\text { D) ouble } \\
\text { Erythema }\end{array}$ & Induration & Necrosis & Blebs \\
\hline \multirow{4}{*}{$\begin{array}{l}\text { Male } \\
\text { Tubcrculin Reaction } \\
\text { in Sizc }\end{array}$} & No. & 328 & 244 & 272 & 39 & 6 \\
\hline & $\%$ & 36.95 & 27.47 & 30.63 & 4.38 & 0.67 \\
\hline & M & 4 & 2 & 5 & 1 & \\
\hline & $M A$ & & 1 & 1 & 1 & \\
\hline \multirow{2}{*}{$\begin{array}{l}\text { Radiological } \\
\text { Suspects }\end{array}$} & Total & 4 & 3 & 6 & 2 & \\
\hline & 泊 & 1.25 & 1.02 & 2.20 & 5.15 & \\
\hline \multirow{4}{*}{$\begin{array}{l}\text { Tuberculin Renction } \\
\text { in Size }\end{array}$} & No. & 157 & 123 & 134 & 28 & 9 \\
\hline & $\therefore$ & 32.00 & 25.01 & 35.39 & 5.69 & 1.84 \\
\hline & $M$ & 3 & 3 & 3 & & \\
\hline & MA & 1 & & 1 & & \\
\hline \multirow{2}{*}{$\begin{array}{l}\text { Rollological } \\
\text { Suspects }\end{array}$} & To!al & 4 & 3 & 4 & & \\
\hline & $\%$ & 2.51 & 2.14 & 2.30 & & \\
\hline
\end{tabular}

다. Af

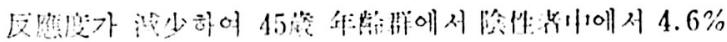

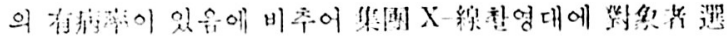

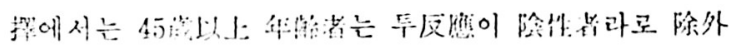

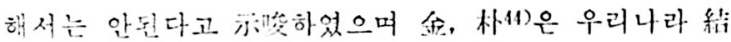
核

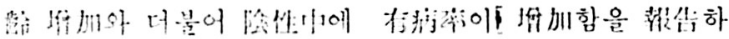

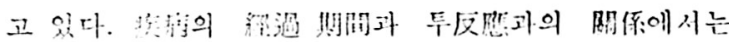

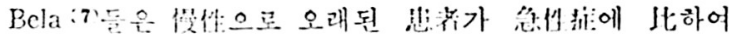

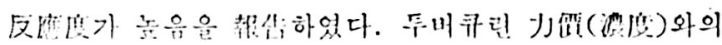

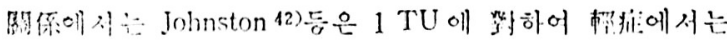

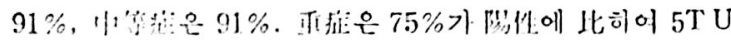

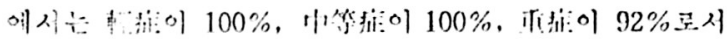

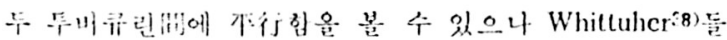
은 $1 \mathrm{TU}$ 에

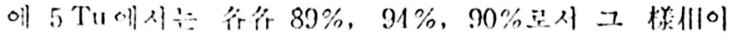

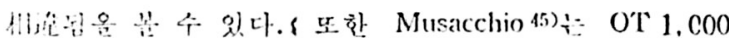

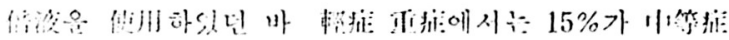

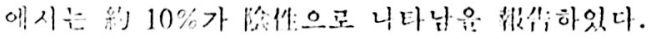

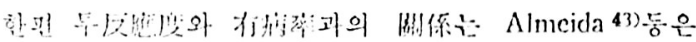

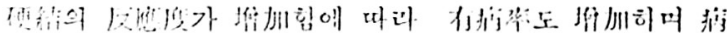

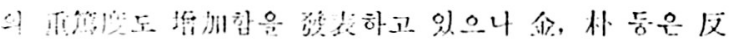

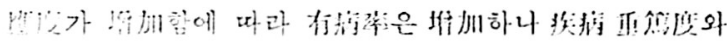

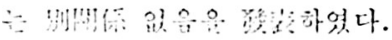

B.C.C. if thend 서 U.S. Public Ilcalth 46) 의

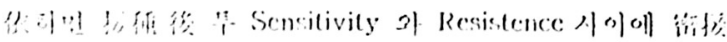

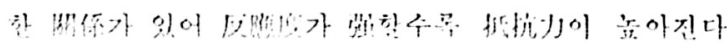

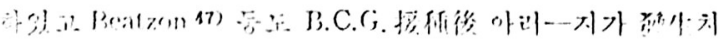

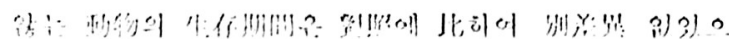

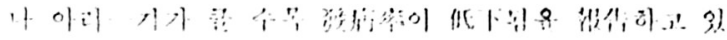

다.

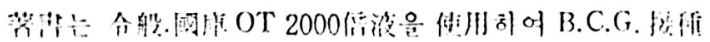

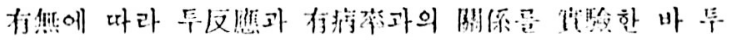

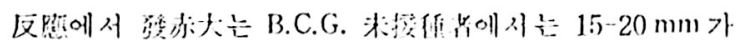

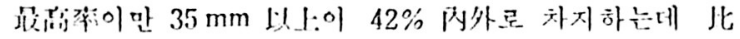
하여 f f f 上이 $20 \%$ 의 不滑하있다.

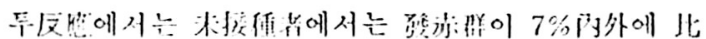

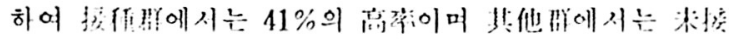

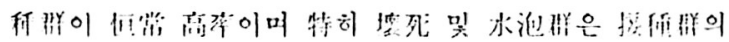
2 (4i 以:이었다.

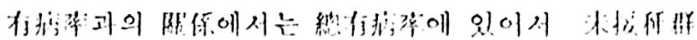

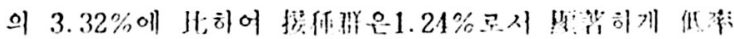
이있 다(P(0.01).

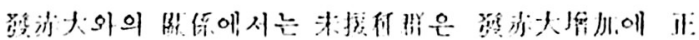

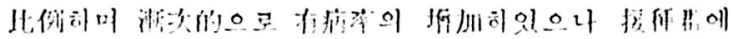

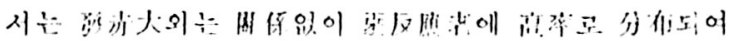

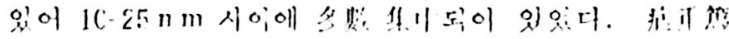

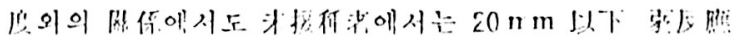

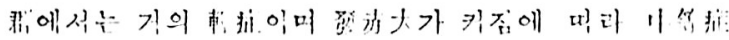

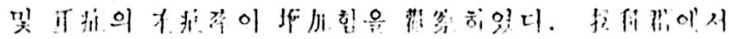

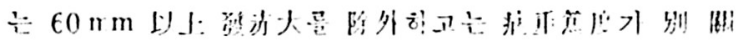
俰불 불 잆있다.

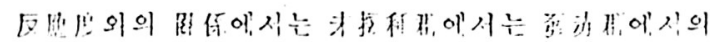

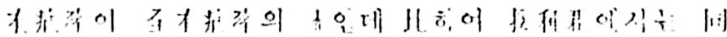

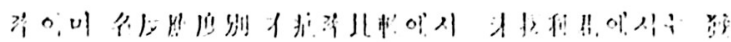

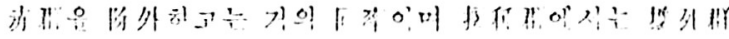

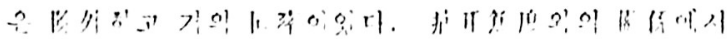

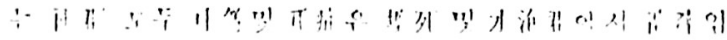
안 


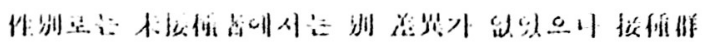

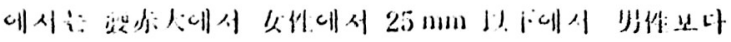

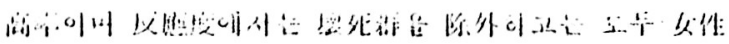

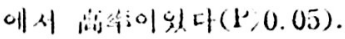

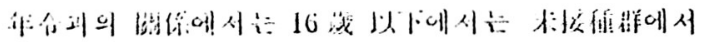

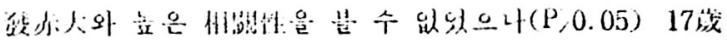

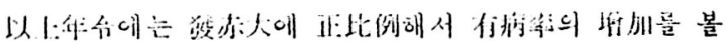

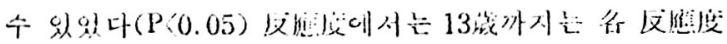

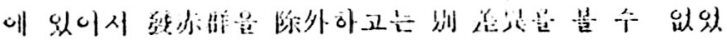

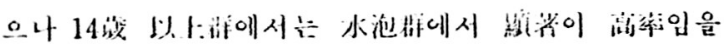
노았다 (P) 0.05).

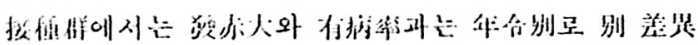

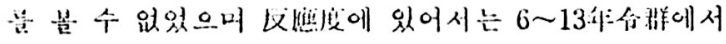

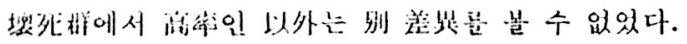

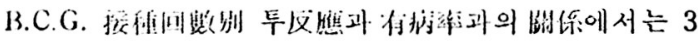

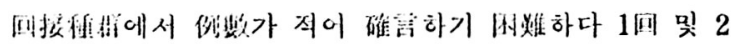

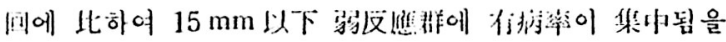

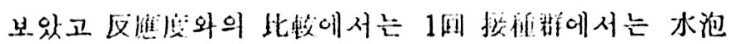

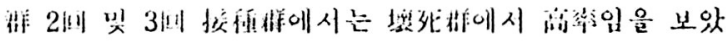
다.

\section{結論}

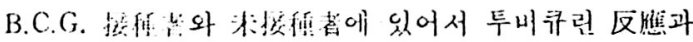

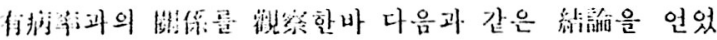
다.

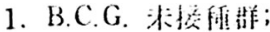

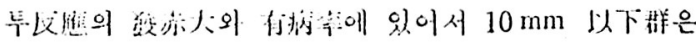

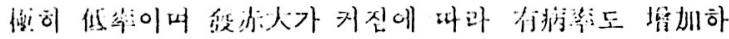

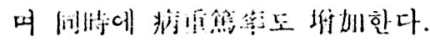

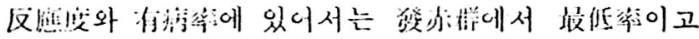

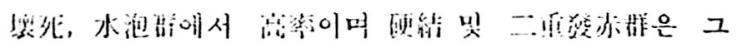

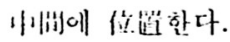

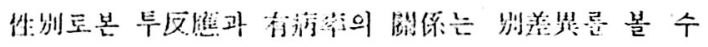
없었다.

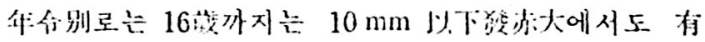

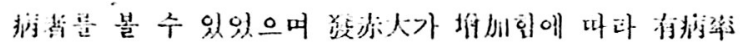

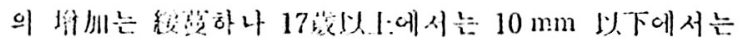

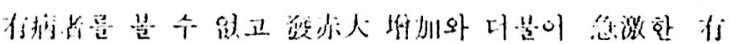

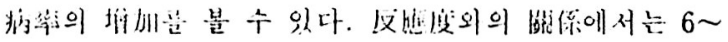

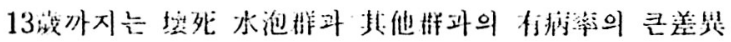

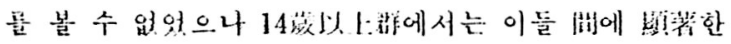

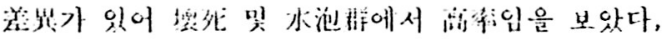

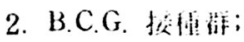

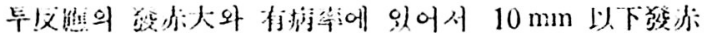

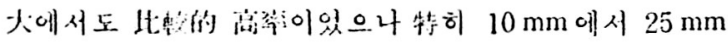
사이가 가장 붕ㅎㅇ이었다.

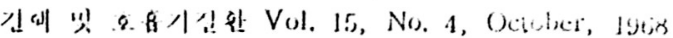

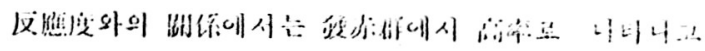

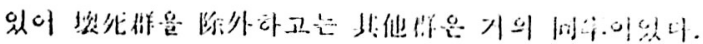

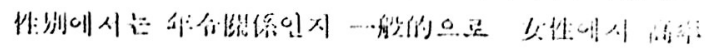

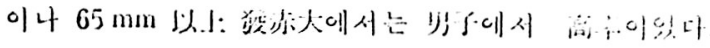

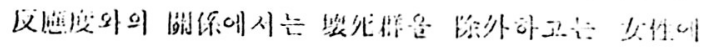
서 齿然이있다.

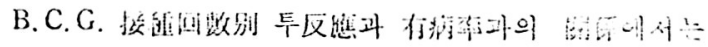

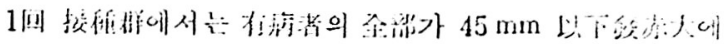

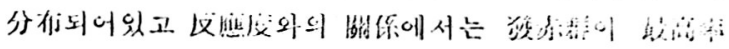
이었다.

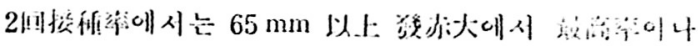

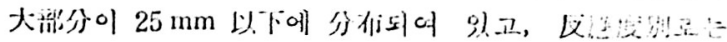

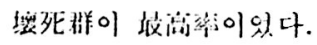

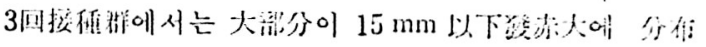

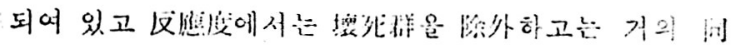
출이있다.

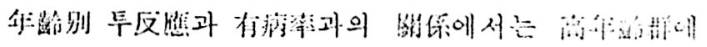

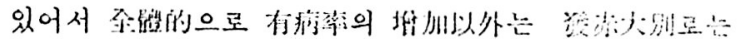

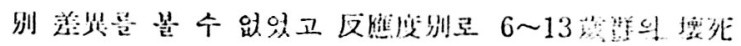

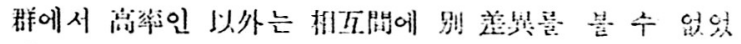
다.

\section{References:}

1) Hasting, E.G., Beach, B.A., and Thompson, I.: The sensitization of cattle to tuberculin by other than tubercle bacilli, Am. Rev. Tuberc., 22:218, 1930.

2) Bruner, S.E.: Eradicating tuberculosis in Pcinsyluania, J.Am. Vet.M.A., 58:147, 1920.

3) Hagan, IV.A.: The no-lesion case problim in the tuberculosis eradication campaign, Cornel! Vat., 21 $: 163,1931$.

4) Long, E.R.: The thlerculin test Its walle and its limitations, 4im.Rev. Taberc., 40:607, 1039.

5) Furcolow, M.L.: Develofinent of calciatation in pulmonary lesions associated with sosititity to Jistoplasmin, Pup. Health Rep., 64:1363. 1949.

6) Iong, E.R.: Tuberculin ancrgy and the varialin!ty of Iubcrculins, Ain. Rov. Tuierc., 39:551. 1939.

7) Carter, R.A.: Pubnonary mectic infocticis, Radiology, $26: 551,1939$.

8) Aronson, J.D., Saylor, R...I, and Park, E.I.:

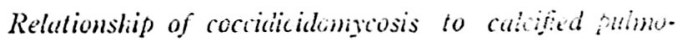
nary nodules, Arch. Path., 3f:31, $19+2$.

9) Palmer, C.E.: Nontuberculuss pulizonary culcification and sensitivity to bistoplasmin, Pub. Heallh Rep., 


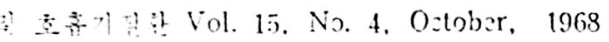

$3045,60.51 .3$.

10) Christic, A., and Peterson, J.C.: Pulmonary calcifi. cation in nesative rators to tubcrulin, Am.J. Pub. Heallh, $: 5: 1131,1045$.

11) Furcolow, M.L.: Development of calcification in Mthmonary tesions associated with scusitivity to histnogsmin, Puh. Health Rep., 64:1363, 1949.

12) Edwatis, L.B. and Krohn, E.F.: Shin scusilirity to antigons mate from varims acidfast bacteria, Am. 1. $H_{1} \mathrm{~s}_{\mathrm{g}}, 66: 253,1957$.

13) Stepinen, V.B.: A study of tuberenlin hypersensitivity in $5: 0$ hts hospilalized for the treatment of active puliannary Lubcreulosis, An. Rev. Tubere., 74:474, 1056.

14) Palmer, C.E., Jablon, S., and Edwards, P.Q.: Thbeculosis morbidity of roung men in relation to lubercilin scnsitivity and body build, Am. Rev. Tuberc., 76:517, 1957.

15) Pepys, J.: The relationship of nonspecific and specific facions in the tubcrulin reaction, Am. Ret. Tubcrc., $73: 49.1055$,

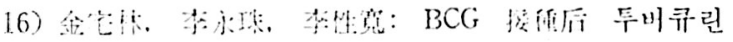

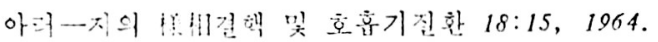

17) Kendig, E.L., Ir.: Unclassificd mycobacteria as a consative ascnt in the positive tubcreulin reaction, Pcdialrics, 30:231, 1962.

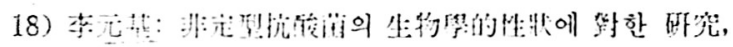
解: $12: 1,1262$.

19) Suthertand, I.A., and Miller, C.L.: Further studies of scusitivity to avian and human old tuberculin in man Tiberle, 45:110, 1964.

20) Mertosin, A., and Eslami, V.: Simullancous comparatire slein tcriing of tuberculous paticn!s with anligens driturd frome M. Iuberculousis and cerlain unclassified mycobarterif. Dis. Chest, 41:624, 1962.

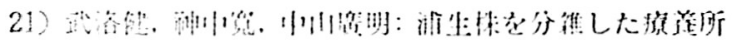

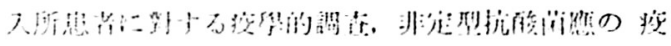

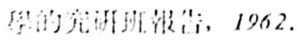

22) Singer, Ea: Non-specific scusitization to $O T$. Asymplomatir infections with mycobacteria, Tubercle. $4 \cdot 2 \% 0,100.5$.

23) Elward, I.P., IIrpwori, I., and Palmer, C.E.: dienifuration of myrobarterial inferlims, Bull. Wha. lilih. ()ris., 33:40.5, 1905.

21 Snith, 1).T., and Johnstom, W.W.: New aspects of

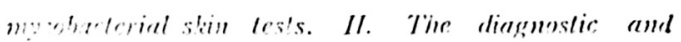

prognostic significanse of qualitative tubercialin test., Arch. Enw. HIth., 10:704. 1965.

25) Kobayashi, Y., and Fukuda, H.: Infmernec of $B C G$ varcination uson skin reactions to purified tuberculo. prolcin cxtracted from unclassified mycobactria, Irla Tubcre. Jap., 14:55, 106.5.

26) Edwards, L.B. and Palmer, C.E.: Epideminlogic studics of tuberculin scnsitivily. II. Response to cxprimental infertion with my obluateria isolated fiom human sourre, Am.I. IJy., 11:218, 1960.

27) Research Committec of the British Tuberculosis Association: Sensitivity to heman and arian tubcrulin among school children in England and Wales, Tubercle,

28) Bater, I.E.: Sonsitivity of humanpopulation to hum. an and avian tuberculin. WHO Aubcrulosis Research Offjec, Bull. Wlit. Hllh. Org. pt. s8:90, 1055.

29) Abrahams, E.W., and Siluestone, H.: Epidemiolngical evidence of the presence of non-tuberculous sensitivily to tuberculin in Quecnsland, Tuberele, 42: 487, $10 \times 1$.

30) Carruthers, K., and Edwards, F.: Atypical mycohaceria in Western Arestralia, Am. Reu. Resp. Dis., 91:887, 1965.

31) Koves-perth, N.: The froblem of Battey discase in Western Australia and a possible rescrvoir, Sond. Bcilrage Klink Tubere, 125:346, 1962.

32) Wong, P.C.: Anomymans mycobacteria isolated from clinical specimen in IInng Kong, J. Trop. Mrd. \& IJyg., 67:100, 1964.

33) Bicrhedal, T.: Comparative sensitieity of school.children in Osin to two mycohacterial antigrns PID.S and PPD.B, Acta Tuberc. Scand., 30:200, 1961.

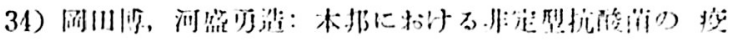

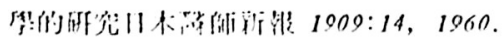

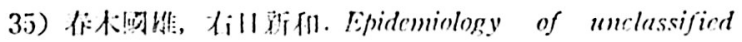

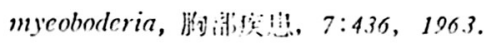

36) Wijsmuller, C.: Naturally arquired tuberculin sensitivily in Nelher lands New Guinea, Bull. Wld. Mllh. Org., 20:641, 19.59

37) Bela O. Dubnczy \& Bernard T. Brown: Amer. Rev. Resp. Dis., 84:69, 1261.

38) Whittuher, J.S.: The tuberdulin test and tuberculosis, Canarl. Med. Asr. J., 82:1222, 1960.

39) Rirh, A.R.: The Pathogenesis of tubcroulesis, Charles C Thomnas Co., Sprinsfirld, Illinois, 1951.

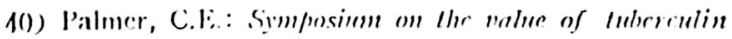


roulians, Ball. Union inlernal. contre laberc., 27:1 106, 1957.

41) British Medical Research Council: $B C C$ and vole bacillus vaccines in the prevention of luberculosis in adolescents, Brit. MI. J., 1:413, 1956.

42) Johnston, W.W., Saltzman, H.A., Bufkin, J.II. and Smith, D.H.: The tuberculin test and the diagnosis of clinical tuberculosis, Amer. Rev. Resp. Dis., 81:1 $89,1960$.

43) Almeida, F Das Neves and Almeida, J.M. Das Neves: Reltion between Degree of tuberculin sensitivity and prevalence of tuberculosis, Bull. Wld. aHth. Org. Vol. 30, 519-528, 1961.

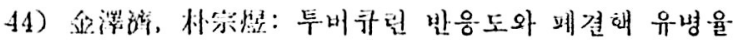

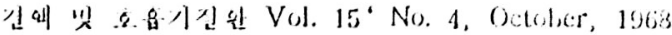

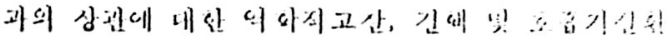
$2: 17,1967$.

45) Musacchio, F.A.: A tuberculin survey of one thousand cases of active tuberculossis, Am. Rev. Tuberc., 42: 120,1940 .

46) : U.S. Public Health Service, Experimcialal stadies of vaccination, allergy, and immunty in tibuculosis, Am. J. Hyts., 62:108, 1955.

47) Jespersen, A. and Bentzon, M.W.: Developincil of tuberculin sensitivity and acouired resistance to tuberculosis in guinea pigs vaccinated with a small dose of BCG vaccine, Acta Path. Micr. Scasld., 5.t: $291,1662$.

-...-Abstract-...-

\title{
STUDY ON RELATION BETWEEN TUBERCULIN REACTION AND RADIOLOGICAL FINDINGS
}

\author{
Lee, Yeung Ju, M.D., Lee, Sung Kwan, M.D.,
}

Dept. of Preventive Medicine, School of Medicine, Kyungpook University

The author has observed both BCG inoculated and non-inoculated group with regard to the results of OT test and X-ray examination coducted upon them.

The summary of the observation is as follows:

1. B.C.G. non-inoculated group

a) In the group less than $10 \mathrm{~mm}$ of erythema, fairly low morbidity rate was noted on $X$-ray examination, while both morbidity and severity rates increased in proportion by the size of erythema,

b) Correlating the intensity of tuberculin reaction with the morbidity rate assuming that suggestive active tuberculosis on $x \cdot$ ray the lowest rate was seen in the erythema group, the highest in the necrosis and bleb groups, and induration and double erythema groups fell on the intermediate level.

c) Sex is found to be no factor in establishing the relationship.

d) By age, the positive findings could be seen in the group under $10 \mathrm{~mm}$ erythema among 16 years old and under. The rate of increase of the positive findings by size of erythema under 16 year of age group was found low in comparison with that of 17 year of age and over group, the latter group showing high increase. However, no positive indings with under $10 \mathrm{~mm}$ erythema were nuted in the latter group.

As to the intensity of the reaction, the morbidity rates were seen almost evenly distributed anong the necrosis, bleb and other groups in under 13 years of age, Over 14 years of age, it was found that morbidity rates were apparently high in the necrosis and bleb groups.

2) B.C.G. inoculated group.

a) Unlike the B.C.G. non-inoculated group, no relationship could be observed between the size of erythema and the morbidity rate. In general, the highest morbidity rate was seen in the group of 11 to $25 \mathrm{~mm}$ of erythema size.

b) As to the intensity of tuberculin reaction vs. the morbidity rates, the highest rate appeared in the erythema group.

In other groups than the necrosis group, the 


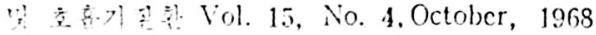

ratos of positive findings were sezh approximately equal, althergh the bleb group showed the lowest among the otiner sroups in intensity, the fact ampacnt! deffering from the non-inoculated group.

c) The higher wate of the positive findings was noted in femir. (xecpt in the crythema group excecding $0.5 \mathrm{~mm}$ in size.

As th the intersity, female showed the higher mete the male except the necrosis group.

d) When the relationship betwen the tuberculin ractios and the morbidity rate under $\mathrm{X}$.ray camaingtion was ricwed from the number of the B.C.G. inoculated, the followings were found:

In the single B.C.G. inoculated group, all the postice findings were distributed in the erythema grous showing less than $45 \mathrm{~mm}$ in size.

is to the intensity of the reaction, the highest mobility rate was scen in the erythema group.

lis the double inoculated group, the highest mondity rate was noted in the erythema group, showing over $65 \mathrm{~mm}$ in size.

However, the positive findings were predomina. ntly distributed in the erythema group under 25 min in size.

$\Lambda$ s to the intensity of the reaction, the highest rate was seen in the necrosis group.

In the triple innculated group, the majority of the positive findings was secn distributed in the erythema genup umeler $15 \mathrm{~mm}$ in size.

The morbility rate were almost cqual among the different intensity gronps except the necrosis.

c) $\Lambda \mathrm{ge} \mathrm{scems}$ on be of $\mathrm{mo}$ factor in establishing the relationship bativen the tuberculin reaction and the morbidity rate, when considered from the erythema size as well as the intensity of the reaction, except for that the age group of 6 to 13 year with necrosis showed higher morbidity rate than the other age groups.

However, it is noted that in higher age brackets the morbidity rate tends to increase in general. 